\title{
Asymptotics and Fast Simulation for Tail Probabilities of Maximum of Sums of Few Random Variables
}

\author{
S. JUNEJA \\ Tata Institute of Fundamental Research, Mumbai \\ R. L. KARANDIKAR \\ Indian Statistical Institute, Delhi \\ and \\ P. SHAHABUDDIN \\ Columbia University, New York
}

\begin{abstract}
We derive tail asymptotics for the probability that the maximum of sums of a few random variables exceeds an increasing threshold, when the random variables may be light as well as heavy tailed. These probabilities arise in many applications including in PERT networks where our interest may be in measuring the probability of large project delays. We also develop provably asymptotically optimal importance sampling techniques to efficiently estimate these probabilities. In the light-tailed settings we show that an appropriate mixture of exponentially twisted distributions efficiently estimates these probabilities. As is well known, exponential twisting based methods are not applicable in the heavy-tailed settings. To remedy this, we develop techniques that rely on "asymptotic hazard rate twisting" and prove their effectiveness in both light and heavy-tailed settings. We show that in many cases the latter may have implementation advantages over exponential twisting based methods in the light-tailed settings. However, our experiments suggest that when easily implementable, the exponential twisting based methods significantly outperform asymptotic hazard rate twisting based methods.
\end{abstract}

Categories and Subject Descriptors: G.3 [Probability and Statistics]: Probabilistic Algorithms (including Monte Carlo); I.6.1 [Simulation and Modeling]: Simulation Theory

General Terms: Algorithms, Performance, Theory

Additional Key Words and Phrases: PERT networks, importance sampling, rare event simulation, tail asymptotics

Authors' addresses: S. Juneja, School of Technology and Computer Science, Tata Institute of Fundamental Research, Colaba, Mumbai, India - 400005; email: juneja@tifr.res.in; R. L. Karandikar, Indian Statistical Institute 7, S J S Sansanwal Marg, New Delhi, India - 110016; email: rlk@isid.ac.in. Permission to make digital or hard copies of part or all of this work for personal or classroom use is granted without fee provided that copies are not made or distributed for profit or direct commercial advantage and that copies show this notice on the first page or initial screen of a display along with the full citation. Copyrights for components of this work owned by others than ACM must be honored. Abstracting with credit is permitted. To copy otherwise, to republish, to post on servers, to redistribute to lists, or to use any component of this work in other works requires prior specific permission and/or a fee. Permissions may be requested from Publications Dept., ACM, Inc., 2 Penn Plaza, Suite 701, New York, NY 10121-0701 USA, fax +1 (212) 869-0481, or permission@acm.org. (C) 2007 ACM 1049-3301/2007/04-ART7 \$5.00 DOI 10.1145/1225275.1225278 http://doi.acm.org/ $10.1145 / 1225275.1225278$

ACM Transactions on Modeling and Computer Simulation, Vol. 17, No. 2, Article 7, Publication date: April 2007. 


\section{ACM Reference Format:}

Juneja, S. Karandikar, R. L., and Shahabuddin, P. 2007. Asymptotics and fast simulation for tail probabilities of maximum of sums of few random variables. ACM Trans. Model. Comput. Simul. 17, 2, Article 7 (April 2007), 35 pages. DOI = 10.1145/1225275.1225278 http://doi.acm.org/ $10.1145 / 1225275.1225278$

\section{INTRODUCTION}

Consider independent random variables $\left(X_{1}, \ldots, X_{n}\right)$ and let $\left(\mathcal{P}_{j}: j \leq \tau\right)$ denote a collection of $\tau$ subsets of $\{1,2, \ldots, n\}$ such that $\cup_{i=1}^{\tau} \mathcal{P}_{j}=\{1,2, \ldots, n\}$. Set

$$
T=\max _{j \leq \tau} \sum_{i \in \mathcal{P}_{j}} X_{i}
$$

In this article, we develop asymptotics and importance sampling techniques for $P(T>u)$ as $u \rightarrow \infty$ for $n$ fixed. When $\tau=1, P(T>u)$ reduces to $P\left(S_{n}>u\right)$ where $S_{n}=\sum_{i=1}^{n} X_{i}$. Also note that the sets $\left(\mathcal{P}_{j}: j \leq \tau\right)$ need not be disjoint so that the associated sums may be dependent.

One application of such probabilities arises in stochastic PERT networks (Project Evaluation and Review Technique; see Elmaghraby [1977] and Adalakha and Kulkarni [1989]). These networks consist of many tasks or activities with stochastic durations that need to be executed under specified precedence constraints. The random variables $\left(X_{1}, \ldots, X_{n}\right)$ may be used to model these task durations and the subsets $\left(\mathcal{P}_{j}: j \leq \tau\right)$ may be chosen to reflect the precedence constraints so that $T$ denotes the overall duration of the project. Our interest then is in efficient estimation of the probability of large delay, $P(T>u)$, in such networks. These probabilities are of enormous interest in project management as the costs associated with large delays can be prohibitive.

Many other applications fit our framework. For example, consider the performance evaluation problem faced by a composite web service provider. As the name suggests, the composite service providers provide a number of services or activities. As in a typical project, these activities may be performed under prespecified precedence constraints. A simple example is the case where a service provider is asked to provide an address of persons $\mathrm{A}$ and $\mathrm{B}$ and the shortest path connecting the two addresses. Another example may be a request to determine the credit rating of a customer where the service provider may need to contact many agencies to establish the customer's credit. Composite service providers in the Internet settings typically provide guarantees to customers against large delays (e.g., delay is less than 5 minutes $95 \%$ of times). Thus, accurate estimation of large delay probabilities is particularly important to them. Our framework may also be used to model grid computing networks where the overall computation consists of many sub-computational processes that may be executed in parallel in different processors but that need to satisfy certain prespecified precedence constraints. Similarly, we may also model transportation schedules in this framework, (e.g., for trains, planes or buses) where precedence constraints are induced by the requirement that a vehicle at a particular location may take off only after the arrival of certain other vehicles. 
Note that when $\tau=n$ and each $\mathcal{P}_{j}=(1,2, \ldots, j)$, then $T=\max _{k \leq n} S_{k}$ so that $P(T>u)$ corresponds to a probability of considerable interest in the applied probability literature.

In our analysis we allow activity durations (i.e., $X_{i}$ 's) to have both light and heavy-tailed distributions (a random variable is said to have a light-tailed distribution if its tail distribution function decays at least at an exponential rate; when the tail distribution function decays at a subexponential rate, under mild regularity conditions, the random variable is said to be subexponentially distributed or heavy tailed; precise definition in Section 2.2). Typically, light-tailed distributions may be used to model activity duration in project management or transportation settings (see Adalakha and Kulkarni [1989]). In the webservices and grid computing settings, heavy-tailed distributions may often be used (see, e.g., Crovella et al. [1998] and Leland et al. [1994]). We further assume that the duration of all the activities are mutually independent, that is, the random variables $\left(X_{1}, \ldots, X_{n}\right)$ are mutually independent.

When the underlying random variables are light tailed, importance sampling probability measure obtained by appropriate exponential twisting of the underlying distributions has been found to be useful for efficient simulation of many performance measures (see survey articles, e.g., Heidelberger [1995] and Juneja and Shahabuddin [2006]; this technique is reviewed in Section 2). In this article, we use and generalize some of these ideas to develop exponential twisting based asymptotically optimal importance sampling techniques to efficiently estimate $P\left(S_{n}>u\right)$ and $P(T>u)$ when the $\left(X_{i}: i \leq n\right)$ are light tailed (asymptotic optimality is a standard criteria for measuring effectiveness of importance sampling distribution and is reviewed in Section 2).

As is well known, an essential requirement to obtain an exponentially twisted distribution from the original distribution of a random variable (rv; rvs when plural) is that it be light tailed (more specifically, its moment generating function should exist in a neighborhood of the origin). When the distribution of the random variable does not satisfy this property (roughly speaking, this is true when the rv is subexponentially distributed) exponential twisting is not feasible. Juneja and Shahabuddin [2002] develop hazard rate twisting to efficiently estimate $P\left(S_{n}>u\right)$ when the $\left(X_{i}: i \leq n\right)$ are independent and identically distributed (iid), and have a subexponential distribution (we review this technique in Section 3.3). In this article, we introduce 'asymptotic hazard rate twisting' in light and heavy-tailed settings. This generalizes the hazard rate twisting proposed for heavy-tailed rvs. In many cases plain hazard rate twisting or exponential twisting (in light-tailed settings) may be difficult to implement, however it may be feasible to successfully implement asymptotic hazard rate twisting.

The specific contributions of this article include:

(1) We develop a logarithmic tail asymptotic for $P(T>u)$ when all the underlying activity durations $X_{i}$ are light tailed, $n$ is fixed and $u \rightarrow \infty$. Enroute, we develop logarithmic asymptotics for the probability $P\left(S_{n}>u\right)$ when the $X_{i}$ 's are light-tailed random variables. Here again, $n$ is fixed and $u \rightarrow \infty$. While there exists enormous literature on logarithmic asymptotics for the 
large deviations probability $P\left(S_{n} / n>u\right)$ as $n \rightarrow \infty$ and $u$ is fixed (see, for example, Dembo and Zeitouni [1998] and Petrov [1975]), to the best of our knowledge, little is documented for the case that we consider (in Jureckova [1981], this problem is considered when $\left(X_{i}: i \leq n\right)$ are iid).

(2) We develop an exact asymptotic for $P(T>u)$ when one or more $X_{i}$ are subexponentially distributed.

(3) We develop exponential twisting based asymptotically optimal importance sampling techniques to efficiently estimate $P\left(S_{n}>u\right)$ when $\left(X_{i}: i \leq n\right)$ are light-tailed. We note through a simple example that straightforward application of exponential twisting may not effectively estimate $P(T>u)$. We then show that an importance sampling distribution that is a convex combination of many appropriately exponentially twisted distributions, asymptotically optimally estimates this probability.

(4) We develop asymptotically optimal importance sampling techniques to estimate $P(T>u)$ (optimal as $u \rightarrow \infty)$ using asymptotic hazard rate twisting in light and heavy-tailed settings.

We restrict our analysis to small networks. Thus, in our analysis of $P(T>u)$, $n$ and hence $\tau$ remain fixed while $u \rightarrow \infty$. This is relevant as in many applications the number of activities may be small (e.g., for composite web services) or an aggregate level analysis may be conducted so that many activities are grouped under a single head resulting in a network consisting of a few aggregated activities. From technical viewpoint, it may be straightforward to generalize exponential-twisting-based methodologies to large $n$ in light-tailed settings. The analysis in Sadowsky and Bucklew [1990] becomes useful here as they consider development of importance sampling techniques for $P\left(S_{n} / n>a\right)$ for $\left(X_{i}: i \leq n\right)$ iid and light tailed, fixed $a>E X_{i}$ and $n \rightarrow \infty$. However, when subexponential random variables are involved, effective importance sampling techniques for estimating $P\left(S_{n} / n>a\right)$ as $n \rightarrow \infty$ do not exist. Also, as we verify through experiments, the hazard twisting techniques discussed here may not be effective for large $n$. Therefore, to maintain focus in this paper we restrict our analysis to fixed $n$ case.

In Section 2, we develop the mathematical background needed to analyze $P(T>u)$. In particular, we review the importance sampling technique and the classification of distributions as light and heavy tailed. In Section 3, we develop logarithmic asymptotics and exponential-twisting-based importance sampling techniques for $P(T>u)$ when all activities are light tailed. In Section 4, we introduce asymptotic hazard rate twisting and we again consider the problem of efficient estimation of $P(T>u)$ when all activities are light-tailed, albeit this time we use asymptotic hazard rate twisting. In Section 5, we develop exact asymptotics and asymptotic hazard rate twisting based importance sampling techniques for $P(T>u)$ when some of the activities are heavy tailed. Some practical implementation considerations related to the proposed importance sampling techniques are discussed in Section 6. Numerical experiments, verifying the efficacy of proposed fast simulation techniques are given in Section 7 . Finally, in Section 8 we provide a brief conclusion and discuss some areas for further research. All proofs are relegated to the appendix. 


\section{MATHEMATICAL FRAMEWORK AND BACKGROUND}

In this section, we first discuss the importance sampling approach in the context of estimating $P(T>u)$ and $P\left(S_{n}>u\right)$. We then classify the distributions as light and heavy tailed and illustrate the definitions through examples.

Let $(\Omega, \mathcal{F}, P)$ denote the underlying probability space on which the mutually independent rvs $\left(X_{1}, \ldots, X_{n}\right)$ are defined. It suffices to let $\mathcal{F}$ correspond to the $\sigma$ algebra generated by $\left(X_{1}, \ldots, X_{n}\right)$.

\subsection{Importance Sampling}

Recall that naive estimation of $P(T>u)$ involves generating many independent samples of $\left(X_{i}: i \leq n\right)$ and hence of $I(T>u)$ via simulation and then taking their average (here $I($.$) denotes the indicator function). As is well known,$ the number of samples needed to get a fixed degree of relative accuracy (ratio of standard deviation and the mean of the estimate) is inversely proportional to $P(T>u)$ and hence becomes prohibitively large for large values of $u$. This motivates the need for importance sampling which we now briefly review: Let $F_{i}$ denote the distribution function of $X_{i}$. To keep the notation simple, we assume that each $X_{i}$ has a probability density function (pdf) given by $f_{i}$. Let $P^{*}$ denote another probability measure on the space $(\Omega, \mathcal{F})$ under which each $X_{i}$ has a distribution function $F_{i}^{*}$ and a pdf $f_{i}^{*}$, such that $P$ is absolutely continuous with respect to $P^{*}$ (i.e., $f_{i}^{*}(x)>0$ if $f_{i}(x)>0$ almost everywhere), and the $X_{i}$ 's are mutually independent under $P^{*}$ as well. Importance sampling under the measure $P^{*}$ involves generating samples of $\left(X_{1}, X_{2}, \ldots, X_{n}\right)$ using $P^{*}$. Then, an unbiased estimator of $P(T>u)$ equals $I(T>u) L$ where

$$
L=\frac{f_{1}\left(X_{1}\right)}{f_{1}^{*}\left(X_{1}\right)} \frac{f_{2}\left(X_{2}\right)}{f_{2}^{*}\left(X_{2}\right)} \ldots \frac{f_{n}\left(X_{n}\right)}{f_{n}^{*}\left(X_{n}\right)} \text { a.s. }
$$

and is referred to as the likelihood ratio. The average of many independent samples of $I(T>u) L$ provides an unbiased estimator of $P(T>u)$. For any probability $\tilde{P}$, let $E_{\tilde{P}}$ denote the associated expectation operator. Note that $E_{P^{*}}\left[L^{2} I(T>u)\right] \geq\left(E_{P^{*}}[L I(T>u)]\right)^{2}=P(T>u)^{2}$ and hence

$$
\limsup _{u \rightarrow \infty} \frac{\log E_{P^{*}}\left[L^{2} I(T>u)\right]}{\log P(T>u)} \leq 2 .
$$

Such a $P^{*}$ is defined to be an asymptotically optimal estimator of $P(T>u)$ iff:

$$
\lim _{u \rightarrow \infty} \frac{\log E_{P^{*}}\left[L^{2} I(T>u)\right]}{\log P(T>u)}=2 .
$$

The discussion above holds for $P\left(S_{n}>u\right)$ as well with $T$ replaced by $S_{n}$.

\subsection{Classification of Distributions Based on Tail Behavior}

2.2.1 Light-Tailed and Superexponential Distributions. Consider a rv $X$ with distribution function $F$ and pdf $f$. Let $\bar{F}$ denote its tail distribution function so that $\bar{F}(x)=1-F(x)$. Let $\Lambda$ denote its hazard function, that is, $\Lambda(x)=-\log \bar{F}(x)$. Since $\bar{F}$ is nonincreasing and $\bar{F}(x) \rightarrow 0$ as $x \rightarrow \infty$, it follows 
that $\Lambda$ is non-decreasing and $\Lambda(x) \rightarrow \infty$ as $x \rightarrow \infty$. We say that $X$ has a lighttailed distribution if there exists a positive extended real number $\lambda \in(0, \infty]$ and an $\alpha \geq 1$ such that

$$
\frac{\Lambda(x)}{x^{\alpha}} \rightarrow \lambda
$$

We say that $X$ is superexponentially distributed if above holds with $\alpha>1$. In this case, we say that its tail distribution decays at a superexponential rate. To keep the exposition simple, we do not consider distributions where the above limit does not exist.

Two functions $f$ and $g$ are said to be asymptotically similar if $\lim _{x \rightarrow \infty} \frac{f(x)}{g(x)}=$ 1 . This is denoted by $f(x) \sim g(x)$. The following examples illustrate some lighttailed distributions:

Example 2.1. Suppose that $X$ is $\operatorname{Gamma}(n, \lambda)$ distributed. Its pdf

$$
f(x)=\frac{\lambda^{n} x^{n-1} \exp [-\lambda x]}{(n-1) !},
$$

for $x>0$ and $f(x)=0$ otherwise, where $n$ is a positive integer and $\lambda>0$. Its tail $\mathrm{df}$ equals

$$
\exp [-\lambda x] \sum_{m=0}^{n-1} \frac{(\lambda x)^{m}}{m !}
$$

Its hazard function is asymptotically similar to $\lambda x$. Hence, it is light tailed but not superexponentially distributed.

Example 2.2. Suppose that $X$ has a Weibull $(\alpha, \lambda)$ distribution, that is, $P(X>x)=\exp \left(-(\lambda x)^{\alpha}\right), x>0$, where $\lambda$ is called the scale parameter and $\alpha$ is called the shape parameter. Then, for $\alpha \geq 1$, it is light tailed and for $\alpha>1$ it is superexponentially distributed. Here the hazard function is $\Lambda(x)=(\lambda x)^{\alpha}$.

Example 2.3. Suppose that $X$ has a Normal distribution with mean $\mu$ and variance $\sigma^{2}$ (let $N\left(\mu, \sigma^{2}\right)$ denote a rv with this distribution). Its tail df $\bar{F}$ evaluated at $x$ equals the tail $\mathrm{df}$ of a $N(0,1)$ rv evaluated at $(x-\mu) / \sigma$. Thus, the following inequalities follow (see, for example, Feller [1970], VII.1, Lemma 2, pg. 175):

$$
\begin{array}{r}
\frac{1}{\sqrt{2 \pi}} \frac{\sigma}{x-\mu}\left(1-\left[\frac{\sigma}{x-\mu}\right]^{2}\right) \exp \left[-\frac{(x-\mu)^{2}}{2 \sigma^{2}}\right]<\bar{F}(x) \\
<\frac{1}{\sqrt{2 \pi}} \frac{\sigma}{x-\mu} \exp \left[-\frac{(x-\mu)^{2}}{2 \sigma^{2}}\right],
\end{array}
$$

for $x \geq \mu$. Although the hazard function $\Lambda$ of $N\left(\mu, \sigma^{2}\right)$ does not have a simple explicit representation, it follows that for any $\epsilon>0$ and $x$ sufficiently large:

$\frac{(x-\mu)^{2}}{2 \sigma^{2}}+\log \left(\frac{x-\mu}{\sigma}\right)+\log (\sqrt{2 \pi}) \leq \Lambda(x) \leq \frac{(x-\mu)^{2}}{2 \sigma^{2}}+\log \left(\frac{x-\mu}{\sigma}\right)+\log (\sqrt{2 \pi})+\epsilon$.

In particular, $\Lambda(x) \sim \frac{(x-\mu)^{2}}{2 \sigma^{2}} \sim \frac{x^{2}}{2 \sigma^{2}}$ and $X$ is superexponentially distributed. 
2.2.2 Heavy-Tailed or Subexponential Distributions. A $\operatorname{rv} X$ is said to be subexponentially distributed if it is nonnegative and if

$$
\frac{P\left(X_{1}+X_{2}>u\right)}{2 P\left(X_{1}>u\right)} \rightarrow 1
$$

as $u \rightarrow \infty$, where $X_{1}$ and $X_{2}$ are independent and have the same distribution as $X$ (see, for example, Embrechts et al. [1997] and Pakes [2004] generalizes this definition to remove the non-negativity restriction. However, we consider only non-negative subexponential random variables in this paper as this considerably simplifies some of our analysis).

It can be shown that if $X$ is subexponentially distributed then its moment generating function evaluated at any positive value equals $\infty$. Furthermore, if the hazard function $\Lambda$ of $X$ is sublinear, that is, $\Lambda(x) / x \rightarrow 0$ as $x \rightarrow \infty$ then under certain regularity conditions $X$ is subexponentially distributed (see Pitman [1980]). For instance, if for all $x$ sufficiently large, $\Lambda(x)=x / \log (x)$, then $X$ is subexponentially distributed.

Consider the following well-known family of subexponential distributions (see, e.g., Embrechts et al. [1997]):

Example 2.4. Suppose that $X$ has a Weibull $(\alpha, \lambda)$ distribution, that is, $P(X>x)=\exp \left(-(\lambda x)^{\alpha}\right), x>0$ with shape parameter $\alpha<1$. Here the hazard function $\Lambda(x)=(\lambda x)^{\alpha}$ is sublinear.

Example 2.5. Suppose that $X=\exp \left[N\left(\mu, \sigma^{2}\right)\right]$, that is, it has a Lognormal distribution. Then, its pdf

$$
f(x)=\frac{1}{x \sqrt{2 \pi \sigma^{2}}} \exp \frac{\left(-(\log x-\mu)^{2}\right.}{\left.2 \sigma^{2}\right)} \quad \text { for } \quad x>0 .
$$

Note that its tail $\mathrm{df}$ evaluated at $x$ equals the tail $\mathrm{df}$ of $N\left(\mu, \sigma^{2}\right)$ evaluated at $\log (x)$. The same therefore is true for the hazard function $\Lambda$. Then, for any $\epsilon>0$ and $x$ sufficiently large:

$$
\frac{(\log (x)-\mu)^{2}}{2 \sigma^{2}}+\log \left(\frac{\log (x)-\mu}{\sigma}\right)+\log (\sqrt{2 \pi}) \leq \Lambda(x),
$$

and

$$
\Lambda(x) \leq \frac{(\log (x)-\mu)^{2}}{2 \sigma^{2}}+\log \left(\frac{\log (x)-\mu}{\sigma}\right)+\log (\sqrt{2 \pi})+\epsilon .
$$

In particular, $\Lambda(x) \sim \frac{(\log (x)-\mu)^{2}}{2 \sigma^{2}}$. It is well known that $X$ is subexponentially distributed (see, e.g., Embrechts et al. [1997]).

Example 2.6. Suppose that $X$ has a $\operatorname{Pareto}(\alpha, \lambda)$ distribution, that is,

$$
P(X>x)=\frac{1}{(1+\lambda x)^{\alpha}} \quad \text { for } \quad x \geq 0 .
$$

Then,

$$
\Lambda(x)=\alpha \log (1+\lambda x) .
$$


In the literature there are different notions of heavy-tailed random variables. Most general classification considers random variable $X$ to be heavy tailed if

$$
\exp [\epsilon x] P(X \geq x) \rightarrow \infty
$$

as $x \rightarrow \infty$ for all $\epsilon>0$. Under this definition, subexponentially distributed random variables are a subclass of heavy-tailed random variables. Certain classifications include random variables with Pareto-type polynomially-decaying tails as heavy, while excluding the random variables with lighter Weibull-type tails. Our analysis in Section 5 focuses on subexponential distributions that includes Pareto as well as Weibull distributions (with shape parameter less than 1). In this article, we refer to subexponential distributions as heavy tailed.

In Sections 3 and 4, we allow the light-tailed random variables to take values in $\Re$ (to accommodate Normally distributed random variables commonly used in PERT analysis). In Section 5, our focus is on subexponential random variables. As indicated earlier, there we restrict all random variables to be nonnegative for ease of analysis.

\section{LIGHT-TAILED ANALYSIS USING EXPONENTIAL TWISTING}

In this section, we consider the case where $\left(X_{i}: i \leq n\right)$ are light tailed. We first review the importance sampling technique of exponential twisting. We then consider $P\left(S_{n}>u\right)$ as this provides insights for analyzing the more general probability $P(T>u)$. Specifically, we develop the logarithmic tail asymptotics for $P\left(S_{n}>u\right)$ and show that appropriate exponential twisting based importance sampling distribution asymptotically optimally estimates $P\left(S_{n}>u\right)$. Through an example we illustrate that simple exponential twisting may not work to asymptotically optimally estimate $P(T>u)$. We then propose a convex combination of exponentially twisted distributions to asymptotically optimally estimate $P(T>u)$. (See, e.g., Sadowsky and Bucklew [1990] for an application of a similar idea in the large deviations settings involving multi-dimensional light-tailed random variables.)

\subsection{Logarithmic Tail Asymptotics and Simulation for $P\left(S_{n}>u\right)$}

Let $\mathcal{H}_{i}(\theta)=\log \int_{-\infty}^{\infty} \exp (\theta x) f_{i}(x) d x$ denote the log-moment generating function of $X_{i}$. For $\theta$ such that $\mathcal{H}_{i}(\theta)<\infty$, let $f_{i}^{\theta}$ denote the pdf obtained by exponentially twisting $f_{i}$ by $\theta$, that is,

$$
f_{i}^{\theta}(x)=\exp \left[\theta x-\mathcal{H}_{i}(\theta)\right] f_{i}(x) .
$$

Let $P_{\theta}$ denote the probability measure under which each $X_{i}$ has a pdf $f_{i}^{\theta}$ and $\left(X_{i}: i \leq n\right)$ are mutually independent. Thus, if $P_{\theta}$ is used to estimate $P\left(S_{n}>u\right)$, the corresponding likelihood ratio $L_{\theta}$ equals

$$
L_{\theta}=\exp \left[-\theta S_{n}+\sum_{i \leq n} \mathcal{H}_{i}(\theta)\right] \text { a.s. }
$$

Let $\lambda_{i}(\cdot)$ and $\Lambda_{i}(\cdot)$ denote the hazard rate and hazard function of $X_{i}$, respectively. Then, $f_{i}(x)=\lambda_{i}(x) \exp \left[-\Lambda_{i}(x)\right]$. As is well known, $\lambda_{i}(x)=\Lambda_{i}^{\prime}(x)=$ $f_{i}(x) / \bar{F}_{i}(x)$, where $\Lambda_{i}^{\prime}(x)$ denotes the derivative of $\Lambda_{i}(x)$. 

as

Note that if $\frac{\Lambda_{i}(x)}{\Lambda_{j}(x)} \rightarrow \infty$ as $x \rightarrow \infty$, then $X_{i}$ has a lighter tail compared to $X_{j}$,

$$
\frac{P\left(X_{i}>x\right)}{P\left(X_{j}>x\right)}=\exp \left(-\Lambda_{i}(x)+\Lambda_{j}(x)\right) \rightarrow 0 .
$$

The following assumption is needed to prove Theorem 3.2:

Assumption 3.1. There exists an $\alpha \geq 1, k: 1 \leq k \leq n$ and constants $\tilde{\lambda}_{i}$, $0<\tilde{\lambda}_{i}<\infty, 1 \leq i \leq k$ such that for all $i \leq k$ :

$$
\lambda_{i}(x) \sim \tilde{\lambda}_{i} \alpha x^{\alpha-1} .
$$

(Note that this implies that

$$
\Lambda_{i}(x) \sim \tilde{\lambda}_{i} x^{\alpha}
$$

see [Feller 1971, VIII.9, Theorem 1(b), pg 281]. Furthermore, if $k<n$, then $\Lambda_{i}(x) / x^{\alpha} \rightarrow \infty$ as $x \rightarrow \infty$, for $k<i \leq n$.

Thus, each of the rvs $\left(X_{i}: i>k\right)$ have a lighter tail distribution compared to the rvs $\left(X_{i}: i \leq k\right)$.

Let

$$
\lambda^{*}=\frac{1}{\left(\sum_{i \leq k} 1 / \tilde{\lambda}_{i}^{\frac{1}{\alpha-1}}\right)^{\alpha-1}},
$$

for $\alpha>1$ and

$$
\lambda^{*}=\min _{i \leq k} \tilde{\lambda}_{i}
$$

for $\alpha=1$. Recall that $E_{\tilde{P}}$ denotes the expectation operator associated with probability $\tilde{P}$.

Theorem 3.2. Under Assumption 3.1,

$$
\lim _{u \rightarrow \infty} \frac{\log P\left(S_{n}>u\right)}{u^{\alpha}}=-\lambda^{*}
$$

and

$$
\lim _{u \rightarrow \infty} \frac{\log E_{P_{\theta_{u}}} L^{2} I\left(S_{n}>u\right)}{u^{\alpha}}=-2 \lambda^{*},
$$

where, for $\alpha>1, \theta_{u} \sim \alpha \lambda^{*} u^{\alpha-1}$, and for $\alpha=1, \theta_{u} \sim \min _{i \leq k} \tilde{\lambda}_{i}$ such that $\mathcal{H}_{i}\left(\theta_{u}\right)=$ $o$ (u) for each $i \leq n$.

From Theorem 3.2 , it follows that $P_{\theta_{u}}$ asymptotically optimally estimates $P\left(S_{n}>u\right)$.

Remark 3.3. For $\alpha>1$, the choice $\theta_{u} \sim \alpha \lambda^{*} u^{\alpha-1}$ is motivated by the fact that the value that minimizes an upper bound $\exp \left[-\theta u+\sum_{i \leq n} \mathcal{H}_{i}(\theta)\right]($ for $\theta>0)$ on the right-hand side in (2) is asymptotically similar to $\alpha \lambda^{*} u^{\alpha-1}$; the latter is given by the solution to the equation

$$
\sum_{i \leq n} \mathcal{H}_{i}^{\prime}(\theta)=u, \quad \theta>0
$$


The analysis supporting this is omitted for brevity. When $\alpha=1$, typically, $\theta_{u}$ can be easily selected so that the conditions in Theorem 3.2 hold. For instance, suppose that $X_{1}$ is exponentially distributed with rate $\eta, X_{2}$ has a Gamma distribution with rate $\eta$ and shape parameter $\alpha$, while all other $\left(X_{i}: i \geq 3\right)$ have lighter tails, for example, they are exponentially distributed with rate $\mu>\eta$. Then, we may set $\theta_{u}=\eta\left(1-\frac{1}{u^{\beta}}\right)$, for any $\beta>0$. For this $\theta_{u}, \mathcal{H}_{1}\left(\theta_{u}\right)=\beta \log u$, $\mathcal{H}_{2}\left(\theta_{u}\right)=\beta \alpha \log u$, while $\mathcal{H}_{i}\left(\theta_{u}\right)=\log \frac{\mu}{\mu-\eta-1 / u^{\beta}}$ for $i \geq 3$. Hence, all $\mathcal{H}_{i}\left(\theta_{u}\right)$ are $o(u)$.

Remark 3.4. The proof of Theorem 3.2 does not require the existence of pdf $f_{i}$, or equivalently, the hazard rate $\lambda_{i}$, for each $i$. It holds even if relation (4) is assumed to hold in lieu of (3) in Assumption 3.1. However, since (3) is useful in simplifying later analysis, we retain Assumption 3.1 in its present form.

\subsection{Logarithmic Tail Asymptotics for $P(T>u)$}

The logarithmic tail asymptotics for $P(T>u)$ follows easily from Theorem 3.2. Some notation is needed for this purpose.

Recall from Assumption 3.1 that, roughly speaking, $\left(X_{i}: i \leq k\right)$ have tails of a similar magnitude that are much heavier than the tails of $\left(X_{i}: i>k\right)$. Let $\tilde{\mathcal{P}}_{j}=\mathcal{P}_{j} \cap\{1,2, \ldots, k\}$. For each $j \leq \tau$ such that $\tilde{\mathcal{P}}_{j}$ is nonempty, when $\alpha>1$, let

$$
\lambda_{j}^{*}=\frac{1}{\left(\sum_{i \in \tilde{\mathcal{P}}_{j}} 1 / \tilde{\lambda}_{i}^{\frac{1}{\alpha-1}}\right)^{\alpha-1}} .
$$

Let $\lambda_{j}^{*}=\min _{i \in \tilde{\mathcal{P}}_{j}} \tilde{\lambda}_{i}$ when $\alpha=1$. Set $\lambda_{j}^{*}$ to $\infty$ if $\tilde{\mathcal{P}}_{j}$ is empty. Let $\mathcal{L}_{j}=\sum_{i \in \mathcal{P}_{j}} X_{i}$.

Theorem 3.5. Under Assumption 3.1,

$$
\lim _{u \rightarrow \infty} \frac{\log P(T>u)}{u^{\alpha}}=-\min _{j \leq \tau} \lambda_{j}^{*} .
$$

Remark 3.6. Thus, in the PERT network terminology, the tail decay rate of $T$ is governed by the tail decay rate of the most likely 'path' $\mathcal{P}_{j}, j \leq \tau$ in the network. Note that when $\alpha=1, \min _{j \leq \tau} \lambda_{j}^{*}$ equals $\min _{j \leq k} \tilde{\lambda}_{j}$. Thus, when the tail distribution function of a rv in the network with the heaviest tail decays at an exponential rate, the decay rate of the network delay $T$ is also exponential with the same rate.

\subsection{Asymptotically Optimal Simulation for $P(T>u)$}

In Example 3.7, we illustrate that under exponential twisting the change of measure that asymptotically optimally estimates $P\left(S_{n}>u\right)$ need not asymptotically optimally estimate $P(T>u)$.

Example 3.7. Suppose that $n=2$ and that $X_{1}$ and $X_{2}$ are iid with $N\left(0, \sigma^{2}\right)$ distribution. Then,

$$
\lim _{u \rightarrow \infty} \frac{\log P\left(X_{i}>u\right)}{u^{2}}=-\frac{1}{2 \sigma^{2}}
$$

ACM Transactions on Modeling and Computer Simulation, Vol. 17, No. 2, Article 7, Publication date: April 2007. 
for $i=1$, 2. In particular, Assumption 3.1 holds with $k=2, \alpha=2, \tilde{\lambda}_{1}=\tilde{\lambda}_{2}=$ $\frac{1}{2 \sigma^{2}}$. Also, $\lambda^{*}=\frac{1}{4 \sigma^{2}}$. Further suppose that $T=\max \left(X_{1}, X_{2}\right)$. It follows from Theorem 3.2 that

$$
\lim _{u \rightarrow \infty} \frac{\log P\left(S_{2}>u\right)}{u^{2}}=-\frac{1}{4 \sigma^{2}}
$$

and

$$
\lim _{u \rightarrow \infty} \frac{\log E_{P_{\theta_{u}}} L_{\theta_{u}}^{2} I\left(S_{2}>u\right)}{u^{\alpha}}=-\frac{1}{2 \sigma^{2}},
$$

where $\theta_{u}=\frac{u}{2 \sigma^{2}}$. (Recall the definition of $P_{\theta}$ from Section 3.1). Again, since $X_{1}$ and $X_{2}$ are iid,

$P(T>u)=1-P\left(X_{1} \leq u\right) P\left(X_{2} \leq u\right)=2 P\left(X_{1}>u\right)-P\left(X_{1}>u\right)^{2} \sim 2 P\left(X_{1}>u\right)$, and it follows that

$$
\lim _{u \rightarrow \infty} \frac{\log P(T>u)}{u^{2}}=-\frac{1}{2 \sigma^{2}}
$$

We now argue that

$$
\liminf _{u \rightarrow \infty} \frac{\log E_{P_{\theta_{u}}} L_{\theta_{u}}^{2} I(T>u)}{u^{2}}>-\frac{1}{\sigma^{2}} .
$$

Hence, while $P_{\theta_{u}}$ asymptotically optimally estimates $P\left(S_{2}>u\right)$, it does not do so for $P(T>u)$. To see (11), note that $\mathcal{H}_{i}(\theta)=\theta^{2} \sigma^{2} / 2$ for $i=1,2$. Hence, $\mathcal{H}_{i}\left(\theta_{u}\right)=\frac{u^{2}}{8 \sigma^{2}}$ for $i=1,2$. Specializing (2) to our example, we get:

$$
L_{\theta_{u}}=\exp \left[-\frac{u}{2 \sigma^{2}}\left(X_{1}+X_{2}\right)+\frac{u^{2}}{4 \sigma^{2}}\right]
$$

almost surely. In particular, it follows that $E_{P_{\theta_{u}}} L_{\theta_{u}}^{2} I(T>u)=E_{P} L_{\theta_{u}} I(T>u)$ is bounded from below by

$$
\exp \left[-\frac{1}{2 \sigma^{2}} u(u+\sqrt{u}+c)+\frac{u^{2}}{4 \sigma^{2}}\right] P\left(X_{1} \in(u, u+\sqrt{u})\right) P\left(0 \leq X_{2} \leq c\right)
$$

for a positive constant $c$. Since $X_{1}$ has a $N\left(0, \sigma^{2}\right)$ distribution, it is easily seen that $\frac{P\left(X_{1} \in(u, u+\sqrt{u})\right)}{P\left(X_{1}>u\right)} \rightarrow 1$ as $u \rightarrow \infty$. Hence,

$$
\lim _{u \rightarrow \infty} \frac{\log P\left(X_{1} \in(u, u+\sqrt{u})\right)}{u^{2}}=-\frac{1}{2 \sigma^{2}} .
$$

Taking logarithm of (12), dividing by $u^{2}$ and taking lim inf as $u \rightarrow \infty$, noting (13), the result can be seen to equal $-\frac{3}{4 \sigma^{2}} u^{2}$ and hence (11) follows.

Similarly, it is easily argued that just by exponentially twisting $X_{1}$ by any parameter and not $X_{2}$, or vice-versa, asymptotic optimality cannot be achieved in this example. This is easily seen by considering the contribution to the second moment by the set where the untwisted component taking values greater than $u$.

In Example 3.7, we illustrated that the exponential-twisted-based change of measure that asymptotically optimally estimates $P\left(S_{n}>u\right)$ may not be as 
effective in estimating $P(T>u)$. A brief intuitive explanation is as follows: It is well known that a change of measure that sufficiently emphasizes the most likely paths to the rare event provides an effective importance sampling estimator for the rare event probability (see, e.g., Juneja and Shahabuddin [2006]). However, since the most likely way the event $\left\{S_{n}>u\right\}$ occurs may differ from the way the event $\{T>u\}$ occurs, an importance sampling estimator that is effective in estimating $P\left(S_{n}>u\right)$ may not be as effective in estimating $P(T>u)$. For instance, in Example 3.7, for large $u$, a likely way for $\left\{S_{2}>u\right\}$ to occur can be roughly seen to correspond to both $X_{1}$ and $X_{2}$ taking values close to $u / 2$, while a likely way for $\{T>u\}$ to occur involves $X_{1}$ exceeding $u$ and $X_{2}$ taking a usual small value (relative to $u$ ), or vice-versa. It can be seen in Example 3.7 that under $P_{\theta_{u}}$ each $X_{i}$ has a $N\left(u / 2, \sigma^{2}\right)$ distribution. This explains its lack of effectiveness in estimating $P(T>u)$ in that example.

We now propose an asymptotically optimal change of measure to estimate $P(T>u)$ that involves taking a convex combination of $\tau$ ( $\tau$ is defined in the Introduction) exponentially twisted changes of measure. It can be seen that this change of measure assigns significant probability to events $\left\{\mathcal{L}_{j}>u\right\}$ for $j \leq \tau$ and $\tilde{\mathcal{P}}_{j}$ nonempty (recall that $\mathcal{L}_{j}=\sum_{i \in \mathcal{P}_{j}} X_{i}$ ), and hence to the event

$$
\{T>u\}=\bigcup_{j \leq \tau}\left\{\mathcal{L}_{j}>u\right\}
$$

When $\tilde{\mathcal{P}}_{j}$ is nonempty, let $P_{\theta_{u_{j}}}$ for $j \leq \tau$, denote a change of measure under which the distribution of each $X_{i}$ for $i \in \mathcal{P}_{j}$ is obtained by exponentially twisting the original distribution by $\theta_{u_{j}}$, where $\theta_{u_{j}} \sim \alpha \lambda_{j}^{*} u^{\alpha-1}$, if $\alpha>1$. When $\alpha=1$, select $\theta_{u_{j}}$ such that $\theta_{u_{j}} \sim \lambda_{j}^{*}$ and $\mathcal{H}_{i}\left(\theta_{u_{j}}\right)=o(u)$ for each $i \in \mathcal{P}_{j}$. Set $\theta_{u_{j}}=0$ if $\tilde{\mathcal{P}}_{j}$ is empty. From Theorem 3.2, it follows that $P_{\theta_{u_{j}}}$ is an asymptotically optimal importance sampling distribution to estimate $P\left(\mathcal{L}_{j}>u\right)$ when $\tilde{\mathcal{P}}_{j}$ is nonempty.

Now consider a probability measure $P^{*}$ for $\left(X_{i}: i \leq n\right)$ such that for any $A \in \mathcal{F}:$

$$
P^{*}(A)=\sum_{j \leq \tau} p_{j} P_{\theta_{u_{j}}}(A),
$$

where $\left(p_{j}>0: j \leq \tau\right)$ and $\sum_{j \leq \tau} p_{j}=1$. Note that the likelihood ratio $L$ of $P$ with respect to $P^{*}$ equals

$$
L=\frac{1}{\sum_{j \leq \tau} p_{j} \exp \left(\theta_{u_{j}} \sum_{i \in \mathcal{P}_{j}} X_{i}-\sum_{i \in \mathcal{P}_{j}} \mathcal{H}_{i}\left(\theta_{u_{j}}\right)\right)} \text { a.s. }
$$

Theorem 3.8. Under Assumption 3.1,

$$
\lim _{u \rightarrow \infty} \frac{\log E_{P^{*}}\left[L^{2} I(T>u)\right]}{u^{\alpha}}=-2 \min _{j \leq \tau} \lambda_{j}^{*} .
$$

From this theorem and (10), it follows that $P^{*}$ asymptotically optimally estimates $P(T>u)$ for any arbitrary $\left(p_{j}>0: j \leq \tau\right)$ such that $\sum_{j \leq \tau} p_{j}=1$. Later in Section 6, we discuss a heuristic for arriving at a good choice for $\left(p_{j}: j \leq \tau\right)$. 


\section{LIGHT-TAILED ANALYSIS USING HAZARD RATE TWISTING}

As mentioned in the Introduction, for a subexponential distribution, exponential twisting is no longer feasible as the moment generating function evaluated at any positive value equals $\infty$. To remedy this, Juneja and Shahabuddin [2002] introduced hazard rate twisting to efficiently estimate $P\left(S_{n}>u\right)$ (recall that $\left.S_{n}=\sum_{i \leq n} X_{i}\right)$ where $\left(X_{i}: 1 \leq i \leq n\right)$ are mutually independent, and are identically and subexponentially distributed. In this and the next section we show that hazard rate twisting can be implemented to efficiently estimate $P(T>u)$ (and hence also $P\left(S_{n}>u\right)$ ) even when all the underlying rvs are allowed to have different distributions and may even be light tailed. A potential drawback of this approach is that both for light and heavy-tailed rvs, this twisting may be difficult to implement as it may be difficult to sample from the hazard rate twisted distribution. Fortunately, in many such cases it may be feasible to twist with a function that is asymptotically similar to the hazard function, such that it is easy to sample from the 'asymptotically hazard rate twisted' distribution and the resultant distribution is asymptotically optimal in estimating the rare event probabilities that we consider.

In this section, we first review the hazard rate twisting technique and introduce the more general asymptotic hazard rate twisting technique when all $\left(X_{i}: i \leq n\right)$ are light tailed. We also prove that this technique asymptotically optimally estimates $P(T>u)$ in the light-tailed settings. In Section 5, we develop the idea of asymptotic hazard rate twisting when one or more $\left(X_{i}: i \leq n\right)$ are subexponentially distributed.

\subsection{Hazard Rate Twisting}

As mentioned in Section 3.1, the pdf $f_{i}(x)$ may be re-expressed as $\lambda_{i}(x) \exp \left[-\Lambda_{i}(x)\right]$, where $\lambda_{i}(\cdot)$ and $\Lambda_{i}(\cdot)$ denote the hazard rate and the hazard function of $X_{i}$, respectively. Then the pdf obtained by hazard rate twisting of the original distribution by an amount $\theta<1$ is given by $h_{i}^{\theta}$, where

$$
h_{i}^{\theta}(x)=\lambda_{i}(x)(1-\theta) \exp \left[-(1-\theta) \Lambda_{i}(x)\right] .
$$

Note that $h_{i}^{\theta}$ has corresponding hazard rate $\lambda_{i}(x)(1-\theta)$ and hazard function $\Lambda_{i}(x)(1-\theta)$ at $x$. For instance, the pdf obtained by hazard rate twisting of the Weibull $(\alpha, \lambda)$ distribution in Examples 2.2 and 2.4 by an amount $\theta$ is Weibull $\left(\alpha, \lambda(1-\theta)^{1 / \alpha}\right)$. Similarly, hazard rate twisting of $\operatorname{Pareto}(\alpha, \lambda)$ distribution in Example 2.6 by $\theta$ results in $\operatorname{Pareto}(\alpha(1-\theta), \lambda)$ distribution. The pdf obtained by hazard rate twisting of $N\left(\mu, \sigma^{2}\right)$ has a more complex form. Note that $\lambda_{i}(x)=f_{i}(x) / \bar{F}_{i}(x)$. Hence, $h_{i}^{\theta}(x)$ may alternatively be expressed as $f_{i}(x)(1-\theta) / \bar{F}_{i}(x)^{\theta}$. In particular, the pdf obtained by hazard rate twisting $N\left(\mu, \sigma^{2}\right)$ by amount $\theta$ has the form

$$
\left(\frac{1}{\sqrt{2 \pi} \sigma}\right)^{1-\theta} \frac{(1-\theta) \exp \left[-\frac{1}{2}\left(\frac{x-\mu}{\sigma}\right)^{2}\right]}{\left(\int_{x}^{\infty} \exp \left[-\frac{1}{2}\left(\frac{y-\mu}{\sigma}\right)^{2}\right] d y\right)^{\theta}} .
$$

To see that hazard rate twisting results in a significantly different distribution compared to exponential twisting note that exponentially twisting a 
Weibull $(\alpha, \lambda)$ distribution is feasible only when $\alpha \geq 1$, and for $\alpha>1$ the resultant distribution is no longer Weibull. As mentioned earlier, exponential twisting a Pareto distribution is not feasible. Furthermore, it is easy to check that $N\left(\mu+\theta \sigma^{2}, \sigma^{2}\right)$ is the distribution obtained when $N\left(\mu, \sigma^{2}\right)$ is exponentially twisted by an amount $\theta$.

Juneja and Shahabuddin [2002] show that hazard rate twisting with $\theta_{u}=$ $1-\frac{n}{\Lambda_{i}(u)}$, asymptotically optimally estimates $P\left(S_{n}>u\right)$ when $X_{i}$ 's are iid and subexponentially distributed (asymptotically as $u \rightarrow \infty$, for a fixed $n$ ). This can be easily seen to be true more generally for $\theta_{u}=1-\frac{1}{\Theta\left(\Lambda_{i}(u)\right)}$. Here, we refer to any non-negative function $a(u)$ as $\Theta(b(u))$, where $b(u)$ is another non-negative function, if there exist constants $0<K_{1}<K_{2}$, and $K_{1} b(u) \leq a(u) \leq K_{2} b(u)$ for all sufficiently large $u$.

\subsection{Conditions on Asymptotic Hazard Functions}

Whenever hazard rate twisting results in a distribution from which it is easy to sample (e.g., Weibull distribution and Pareto distribution discussed at the end of Section 4.1), it may be practical to conduct hazard rate twisting to estimate $P(T>u)$. However, when this is not the case, more general methods may be needed. For instance, it may be seen from (16) that hazard rate twisting a Normal distribution does not result in another Normal distribution. In fact it results in a distribution from which generating random variates may be difficult. The same can be seen to be true for the Lognormal distribution. Fortunately, as noted in Example 2.3, the hazard function $\Lambda(x)$ of a Normal distribution is asymptotically similar to $\frac{(x-\mu)^{2}}{2 \sigma^{2}}$. If this function is used to twist the Normal distribution, then we get another Normal distribution. Similarly, from Example 2.5 , it can be seen that if we twist a Lognormal distribution with $\frac{(\log x-\mu)^{2}}{2 \sigma^{2}}$ (asymptotically similar to its hazard function), we again get a Lognormal distribution. We now describe some conditions imposed on the asymptotic hazard functions that, with a suitably selected twisting parameter, ensure that the associated importance sampling asymptotically optimally estimates $P(T>u)$.

Consider non-negative 'asymptotic hazard' functions $\tilde{\Lambda}_{i}(\cdot): \Re \rightarrow \Re^{+}, i \leq k$ such that

$$
\tilde{\Lambda}_{i}(x) \sim \Lambda_{i}(x) .
$$

Further suppose that $\tilde{\Lambda}_{i}(x)$ satisfy the condition

$$
\tilde{\Lambda}_{i}(x) \leq \Lambda_{i}(x)+a_{i} \max \left(\log \Lambda_{i}(x), 1\right)
$$

for some $a_{i} \geq 0$, and for all $x$ and $i \leq k$.

Clearly, the conditions (17) and (18) are satisfied by $\tilde{\Lambda}_{i}(x)=\Lambda_{i}(x)$.

Let $\Gamma_{i}(\theta)=\log \int_{-\infty}^{\infty} \exp \left[\theta \tilde{\Lambda}_{i}(x)\right] f_{i}(x) d x$. Note that if $\tilde{\Lambda}_{i}$ is used as a proxy for $\Lambda_{i}$ in hazard rate twisting of $X_{i}$ by amount $\theta$, the resultant pdf equals

$$
\exp \left[\theta \tilde{\Lambda}_{i}(x)-\Gamma_{i}(\theta)\right] f_{i}(x) .
$$

Also note that $\Gamma_{i}(\theta)=\frac{1}{1-\theta}$ if $\tilde{\Lambda}_{i}=\Lambda_{i}$. 


\subsection{Asymptotically Optimal Simulation}

Consider an importance sampling distribution $P^{*}$ under which all $X_{i}$ 's remain mutually independent. Each $X_{i}$ for $i \leq k$, has a pdf given by

$$
f_{i}^{*}(x)=\exp \left[\theta_{u} \tilde{\Lambda}_{i}(x)-\Gamma_{i}\left(\theta_{u}\right)\right] f_{i}(x)
$$

and $\theta_{u}=1-1 / \Theta\left(u^{\alpha}\right)$. Each $X_{i}$ for $i>k$ has the original distribution.

Note that the likelihood ratio of $P$ with respect to $P^{*}$ equals

$$
L=\exp \left[-\theta_{u} \sum_{i=1}^{k} \tilde{\Lambda}_{i}\left(X_{i}\right)+\sum_{i=1}^{k} \Gamma_{i}\left(\theta_{u}\right)\right] \text { a.s. }
$$

Theorem 4.1. Under Assumption 3.1, (17) and (18),

$$
\lim _{u \rightarrow \infty} \frac{\log E_{P^{*}} L^{2} I(T>u)}{u^{\alpha}}=-2 \min _{j \leq \tau} \lambda_{j}^{*}
$$

In view of Theorem 3.5, this establishes that $P^{*}$ estimates $P(T>u)$ asymptotically optimally. Also note that Theorem 4.1 implies that,

$$
\lim _{u \rightarrow \infty} \frac{\log E_{P^{*}} L^{2} I\left(S_{n}>u\right)}{u^{\alpha}}=-2 \lambda^{*} .
$$

Remark 4.2. In light of Example 3.7, this result may appear surprising as that example illustrates that the exponential twisting based importance sampling distribution that asymptotically optimally estimates $P\left(S_{n}>u\right)$ may not be as efficient in estimating $P(T>u)$. The intuitive explanation for effectiveness of asymptotic hazard rate twisting for both the probabilities is that under this twisting, the probability of a rv taking large values is much larger than that under naive simulation and is typically more spread out compared to exponential twisting. For example, it can be seen that if an asymptotic hazard function $\frac{1}{2}\left(\frac{x-\mu}{\sigma}\right)^{2}$ is used to twist $N\left(\mu, \sigma^{2}\right) \mathrm{rv}$ and $\theta_{u}$ is set to $1-\frac{c}{u^{2}}$ for a constant $c$, then, the resulting distribution is $N\left(\mu, u^{2} \sigma^{2} / c\right)$. It is easy to see that $P\left(N\left(\mu, u^{2} \sigma^{2} / c\right)>u / 2\right)$ and $P\left(N\left(\mu, u^{2} \sigma^{2} / c\right)>u\right)$ both converge to positive constants as $u \rightarrow \infty$.

Remark 4.3. Empirically we observed that the above hazard rate twisting distribution gives large variance reduction compared to naive simulation only when the probability of interest is extremely small. A significant improvement over it is achieved if in a manner analogous to that described in Section 3.3, we use a convex combination of $\tau$ probability measures each using asymptotic hazard rate twisting geared to a particular path as described above (again, the original measure may be used for paths that do not contain any activity $X_{i}$, $i \leq k$ ). The proof of asymptotic optimality then follows exactly as the proof of Theorem 3.8 (with Theorem 4.1 replacing Theorem 3.2 in the proof) and is therefore omitted. In our experiments we report the results using this mixture of probability measures.

\section{HEAVY-TAILED ANALYSIS}

We now develop an exact tail-asymptotic and asymptotic hazard rate twisting based simulation methods to estimate $P(T>u)$ for the case 
where the heaviest-tailed $\mathrm{rv}$ in the network has a subexponential distribution.

\subsection{Exact Asymptotic}

Theorem 5.2 develops an exact asymptotic for $P(T>u)$. The following assumption is needed to prove it.

Assumption 5.1. The random variables $\left(X_{i}: i \leq n\right)$ are non-negative. Furthermore, $X_{1}$ is subexponentially distributed and there exists $a(k: 1 \leq k \leq n)$ and positive constants $\left(\gamma_{i} \leq 1: i \leq k\right)$ such that for $i \leq k$,

$$
\lim _{u \rightarrow \infty} \frac{P\left(X_{i}>u\right)}{P\left(X_{1}>u\right)}=\gamma_{i}
$$

and

$$
\lim _{u \rightarrow \infty} \frac{P\left(X_{i}>u\right)}{P\left(X_{1}>u\right)}=0
$$

for $i>k($ if $k<n)$.

Under Assumption 5.1, it is well known that

$$
P\left(\sum_{i \leq n} X_{i}>u\right) \sim P\left(\max _{i \leq n} X_{i}>u\right) \sim \sum_{i \leq n} P\left(X_{i}>u\right),
$$

(see, e.g., Sigman [1999]).

Theorem 5.2. Under Assumption 5.1,

$$
P(T>u) \sim\left(\sum_{i=1}^{k} \gamma_{i}\right) P\left(X_{1}>u\right) .
$$

Example 5.3. Consider the case where all $X_{i}$ 's have a Weibull distribution, that is, $P\left(X_{i}>x\right)=\exp \left(-\left(\eta_{i} x\right)^{\alpha_{i}}\right), x>0$. Suppose that $\alpha_{1}<1$ so that $X_{1}$ is subexponentially distributed (see, e.g., Embrechts et al. [1997]). Since it has the heaviest tail, it follows that if $\alpha_{i}=\alpha_{1}$ then, $\eta_{i} \geq \eta_{1}$. In this setting, note that

$$
\lim _{u \rightarrow \infty} \frac{P\left(X_{i}>u\right)}{P\left(X_{1}>u\right)}
$$

equals either one or zero. Hence, $k$ corresponds the number of rvs with the distribution identical to $X_{1}$, and $P(T>u) \sim k P\left(X_{1}>u\right)=k \exp \left(-\left(\eta_{1} u\right)^{\alpha_{1}}\right)$.

Similarly, if for each $i, X_{i}$ has a Lognormal $\left(\mu_{i}, \sigma_{i}^{2}\right)$ distribution, that is, its pdf

$$
f_{i}(x)=\frac{1}{x \sqrt{2 \pi \sigma_{i}^{2}}} \exp \left(\frac{-\left(\log x-\mu_{i}\right)^{2}}{2 \sigma_{i}^{2}}\right) \quad \text { for } \quad x>0,
$$

then again $\sigma_{i}^{2} \leq \sigma_{1}^{2}$ and if $\sigma_{i}^{2}=\sigma_{1}^{2}$, then $\mu_{i} \leq \mu_{1}$. Here again, $k$ denotes the number of rvs with the distribution identical to $X_{1}$, and

$$
P(T>u) \sim k P\left(X_{1}>u\right) \sim \frac{k \sigma_{1}}{\sqrt{2 \pi} \log u} \exp \left(\frac{-\left(\log u-\mu_{1}\right)^{2}}{\left(2 \sigma_{1}^{2}\right)}\right)
$$

(an exact asymptotic of $P\left(X_{1}>u\right)$ may be inferred from Example 2.3). 
Finally, suppose that all $X_{i}$ have a $\operatorname{Pareto}\left(\alpha_{i}, \eta_{i}\right)$ distribution, that is,

$$
P\left(X_{i}>x\right)=\frac{1}{\left(1+\eta_{i} x\right)^{\alpha_{i}}} \quad \text { for } \quad x>0 .
$$

Now $X_{i}$, for $i \leq k$ are rvs with $\alpha_{i}=\alpha_{1}$, while $\alpha_{i}>\alpha_{1}$ for $i>k$. Hence,

$$
P(T>u) \sim \sum_{i=1}^{k} \frac{1}{\left(1+\eta_{i} u\right)^{\alpha_{1}}} \sim \frac{1}{u^{\alpha_{1}}} \sum_{i=1}^{k} \frac{1}{\eta_{i}^{\alpha_{1}}} .
$$

\subsection{Conditions on Asymptotic Hazard Functions}

We now describe the conditions imposed on the non-negative asymptotic hazard functions $\tilde{\Lambda}_{i}(\cdot): \Re \rightarrow \Re^{+}, i \leq n$, that can be used to asymptotically optimally estimate $P(T>u)$ via appropriate importance sampling. As in the light-tailed settings, we assume that

$$
\tilde{\Lambda}_{1}(x) \sim \Lambda_{1}(x),
$$

and for all $i$, there exists $a_{i} \geq 0$,

$$
\tilde{\Lambda}_{i}(x) \leq \Lambda_{i}(x)+a_{i} \max \left(\log \Lambda_{i}(x), 1\right) .
$$

Rather than selecting $\tilde{\Lambda}_{i}(u) \sim \Lambda_{i}(u)$ for $i \geq 2$, as in the light-tailed settings, we allow greater flexibility in the choice of $\tilde{\Lambda}_{i}$ by considering those that satisfy

$$
\liminf _{u \rightarrow \infty} \frac{\tilde{\Lambda}_{i}(u)}{\tilde{\Lambda}_{1}(u)} \geq 1
$$

for $i \geq 2$. It is easy to see that the Assumption 5.1 ensures that the hazard functions satisfy this condition. To see this, note that under Assumption 5.1,

$$
\lim _{u \rightarrow \infty} \Lambda_{1}(u)\left(\frac{\Lambda_{i}(u)}{\Lambda_{1}(u)}-1\right) \geq 0
$$

Therefore,

$$
\liminf _{u \rightarrow \infty} \frac{\Lambda_{i}(u)}{\Lambda_{1}(u)} \geq 1
$$

To ease the analysis, we further restrict $\tilde{\Lambda}_{1}$ to be eventually a nondecreasing function and to satisfy the following property: For every $\epsilon>0$, there exists a $u_{\epsilon}$ such that

$$
\sum_{i=1}^{n} \tilde{\Lambda}_{1}\left(x_{i}\right) \geq \tilde{\Lambda}_{1}\left(\sum_{i=1}^{n} x_{i}\right)-\epsilon .
$$

for all $\left(x_{1}, \ldots, x_{n}\right) \geq 0$ with $\sum_{i=1}^{n} x_{i} \geq u_{\epsilon}$. This property is shown to be true in Juneja and Shahabuddin [2002] for any non-negative function $\Lambda(\cdot)$ that is eventually everywhere differentiable and whose derivative is eventually decreasing to zero. It is satisfied by hazard rates of commonly encountered subexponential distributions such as Pareto, Lognormal and Weibull distributions with shape parameter less than 1.

ACM Transactions on Modeling and Computer Simulation, Vol. 17, No. 2, Article 7, Publication date: April 2007. 
As mentioned in Section 4, for Lognormal $\left(\mu_{i}, \sigma_{i}^{2}\right)$ rv, the hazard-rate-twisted distribution is not Lognormal and may be difficult to sample from. However, $\Lambda_{i}(x) \sim\left(\log x-\mu_{i}\right)^{2} /\left(2 \sigma_{i}^{2}\right)$. Hence, we can set $\tilde{\Lambda}_{i}(x)=\left(\log x-\mu_{i}\right)^{2} /\left(2 \sigma_{i}^{2}\right)$. This function satisfies the restrictions imposed on $\tilde{\Lambda}_{i}(\cdot)$ in (25) and the resultant twisted distribution can be seen to be Lognormal.

\subsection{Asymptotically Optimal Simulation}

Let $P^{*}$ denote the probability measure under which the $X_{i}$ 's remain mutually independent and each $X_{i}$ has the distribution

$$
d F_{i}^{*}(x)=\exp \left[\theta_{u} \tilde{\Lambda}_{i}(x)-\Gamma_{i}\left(\theta_{u}\right)\right] d F_{i}(x),
$$

where $\theta_{u}=1-1 / \Theta\left(\tilde{\Lambda}_{1}(u)\right)$. In Juneja and Shahabuddin [2002], this value of $\theta_{u}$ is shown to work well for estimating $P\left(S_{n}>u\right)$ when all rvs are iid and subexponentially distributed and one uses plain hazard rate twisting. The fact that $P(T>u)$ has the same exact asymptotic as $P\left(S_{n}>u\right)$ suggests that a similar $\theta_{u}$ may work in this setting as well.

Theorem 5.2 implies that

$$
\lim _{u \rightarrow \infty} \frac{\log P(T>u)}{\Lambda_{1}(u)}=-1 .
$$

Theorem 5.4 states the main result of this section. Together with (28), it implies asymptotic optimality of $P^{*}$.

Theorem 5.4. Under Assumption 5.1, (24), (25) and (27),

$$
\lim _{u \rightarrow \infty} \frac{\log E_{P^{*}} L^{2} I(T>u)}{\Lambda_{1}(u)}=-2 .
$$

Remark 5.5. Theorem 5.4 can be seen to hold even if the light-tailed $X_{i}^{\prime} s$ retain their original distribution under $P^{*}$. The proof then follows along the lines of Theorem 3.2 where all rvs with $\alpha_{i}>\alpha$ retain their original distribution under the asymptotically optimal change of measure. However, we do not explicitly prove this to avoid repetition.

Remark 5.6. As we discussed in Remark 4.3 in the light-tailed settings, significant performance improvement over $P^{*}$ may be achieved, if in a manner analogous to that described in Section 3.3, we use a convex combination of $\tau$ probability measures each using asymptotic hazard rate twisting geared to a particular path. The proof of asymptotic optimality then follows exactly as the proof of Theorem 3.8. As in the light-tailed settings, in our experiments we report the results using this mixture of probability measures.

\section{PARAMETER SELECTION FOR IMPORTANCE SAMPLING}

In the previous sections we gave order of magnitude specifications of optimal $\theta_{u}$ for asymptotically optimal estimation of $P\left(S_{n}>u\right)$ and $P(T>u)$. Similarly, we proved that any choice of $\left(p_{j}: j \leq \tau\right)$ where each $p_{j}>0$ and $\sum_{j \leq \tau} p_{j}=1$ can be used to asymptotically optimally estimate $P(T>u)$ with appropriate 
probability measures corresponding to each set $\mathcal{P}_{j}$. Empirically, we observe that when superexponential rvs are involved and exponential twisting is conducted in estimating $P\left(S_{n}>u\right)$, setting $\theta_{u}$ as a solution to (8) performs very well.

Empirically we also observe that when asymptotic hazard rate twisting or hazard rate twisting is applied, setting $\theta_{u}$ to any $1-1 / \Theta\left(u^{\alpha}\right)$ function in lighttailed settings (Theorem 4.1) and $1-1 / \Theta\left(\Lambda_{1}(u)\right)$ function in the heavy-tailed settings (Theorem 5.4) may achieve variance reduction only for extremely large values of $u$. To achieve simulation efficiency increase for practical ranges of $u$, say when the rare event probabilities are of the order of $10^{-1}$ to $10^{-6}$, we need good heuristics to select $\theta_{u}$. Similarly, though setting each $p_{j}=1 / \tau$ gives reliable results, the results are improved substantially (about 2-3 times in our experiments) by selecting them cleverly, as outlined in this section.

\subsection{Selecting $\theta_{u}$}

We specialize a heuristic from Huang and Shahabuddin [2004] that considers a more general set-up. We focus on arriving at a good $\theta_{u}$ for estimating $P\left(S_{n}>u\right)$. This then extends to finding $\operatorname{good}\left(\theta_{u_{j}}, j \leq \tau\right)$ for estimating $P(T>u)$ by setting each $\theta_{u_{j}}$ to the value that is good for estimating the corresponding $P\left(\mathcal{L}_{j}>u\right)$.

For estimating $P\left(S_{n}>u\right)$, the second moment obtained by asymptotic hazard rate twisting by amount $\theta$ is given by (see (19))

$$
E_{P^{*}}\left(L^{2} I\left(S_{n}>u\right)\right)=\exp \left(2 \sum_{i \leq n} \Gamma_{i}(\theta)\right) E_{P^{*}}\left[\exp \left(-2 \theta \sum_{i \leq n} \tilde{\Lambda}_{i}\left(X_{i}\right)\right) I\left(S_{n}>u\right)\right],
$$

both under light and heavy-tailed settings. Here, for light-tailed settings, we have assumed that $k$ defined in Assumption 3.1 equals $n$. When, $k<n$, the impact of $\left(X_{i}: i>k\right)$ on the rare event probability $P\left(S_{n}>u\right)$ is negligible, so heuristically we can proceed with finding best twisting parameter by ignoring these rvs.

We now follow two steps. In the first step, for a given $\theta \geq 0$, we find a deterministic upper bound on $E_{P^{*}}\left(L^{2} I\left(S_{n}>u\right)\right)$. This is done by minimizing $\sum_{i \leq n} \tilde{\Lambda}_{i}\left(x_{i}\right)$ over all $\left(x_{i}: i \leq n\right)$ satisfying $\sum_{i \leq n} x_{i} \geq u$. In the case where any random variable $X_{i}$ is non-negative we add an additional constraint $x_{i} \geq 0$. Let $g(u)$ denote such a solution. This yields the bound

$$
E_{P^{*}}\left(L^{2} I\left(S_{n}>u\right)\right) \leq \exp \left(2 \sum_{i \leq n} \Gamma_{i}(\theta)-2 \theta g(u)\right) .
$$

Often, $g(u)$ may not be available in closed form, however a simple approximate solution to $g(u)$ may be found that is close to it for all sufficiently large values of $u$ (this is illustrated through an example in Case 1 in Section 7). Then, we replace $g(u)$ above by its approximation. In the second step we find the $\theta=\theta_{u} \geq 0$ that minimizes the above bound. In all the examples reported in Section 7 , the $\theta_{u_{j}}$ so obtained (by focussing on $P\left(\mathcal{L}_{j}>u\right)$ ) have a form that is consistent with the requirements in the corresponding theorem statements, thus assuring asymptotic optimality. For small $u$, the $\theta_{u_{j}}$ so obtained may be negative. Then, we set $\theta_{u_{j}}=0$, corresponding to naive simulation. 


\subsection{Selection of $p_{j}$ 's}

Here, we use the ideas reported in Juneja and Shahabuddin [2006]. We illustrate them in the framework in Section 3.3. Again, first an upper bound to $E_{P^{*}}\left(L^{2} I(T>u)\right)$ is developed where $L$ is given by (14). Then, we find the vector $\left(p_{j}: j \leq \tau\right)$ that minimizes this bound. Note that for $\theta_{u_{j}}>0$,

$$
L I\left(\mathcal{L}_{j}>u\right) \leq \frac{K_{j}}{p_{j}} I\left(\mathcal{L}_{j}>u\right),
$$

where $K_{j}=\exp \left(-\theta_{u_{j}} u+\sum_{i \in \mathcal{P}_{j}} \mathcal{H}_{i}\left(\theta_{u_{j}}\right)\right)$. Therefore,

$$
L I(T>u) \leq\left(\max _{j \leq \tau} \frac{K_{j}}{p_{j}}\right) I(T>u),
$$

and

$$
E_{P^{*}}\left(L^{2} I(T>u)\right) \leq\left(\max _{j \leq \tau} \frac{K_{j}}{p_{j}}\right)^{2} .
$$

Minimizing the above upper bound subject to $\sum_{j \leq \tau} p_{j}=1$, and $p_{j} \geq 0$ for $j \leq \tau$, yields the weights

$$
p_{j}^{*}=\frac{K_{j}}{\sum_{i=1}^{m} K_{i}} .
$$

The asymptotic hazard rate twisting case, both in light and heavy-tailed settings, is similar; in this case we get the same expression for $p_{j}^{*}$ except that now $K_{j}=\exp \left(-\theta_{u_{j}} g(u)+\sum_{i \in \mathcal{P}_{j}} \Gamma_{i}\left(\theta_{u_{j}}\right)\right)$. Here, we make use of the bound in (31) evaluated at $\theta=\theta_{u_{j}}$. This methodology is used to determine $p_{j}$ 's in the experiments in Section 7.

\section{NUMERICAL RESULTS}

We consider an example with $n=15$ and $\tau=10$. The $\left(\mathcal{L}_{j}: j \leq 10\right)$, respectively, equal

$$
\begin{gathered}
X_{1}+X_{4}+X_{11}+X_{15}, \\
X_{1}+X_{4}+X_{12}, \\
X_{2}+X_{5}+X_{11}+X_{15}, \\
X_{2}+X_{5}+X_{12}, \\
X_{2}+X_{6}+X_{13}, \\
X_{2}+X_{7}+X_{14}, \\
X_{3}+X_{8}+X_{11}+X_{15}, \\
X_{3}+X_{8}+X_{12}, \\
X_{3}+X_{9}+X_{15}, \\
\text { and } X_{3}+X_{10}+X_{14} .
\end{gathered}
$$

We test the performance of the proposed techniques for five sets of distributions of $\left(X_{i}: i \leq 15\right)$ described below. For $j \leq 10$, let $n_{j}$ denote the number of terms in $\mathcal{P}_{j}$.

ACM Transactions on Modeling and Computer Simulation, Vol. 17, No. 2, Article 7, Publication date: April 2007. 
Case 1. Subexponential-tailed with Hazard Rate Twisting. In this case $X_{1}, \ldots, X_{8}$ have the Pareto $(2,1)$ distribution and $X_{9}, \ldots, X_{15}$ have the Weibull $(0.5,2)$ distribution. Note that in this case all $X_{i}$ 's are heavy tailed. We set $\tilde{\Lambda}_{i}(x)=\Lambda_{1}(x)=2 \ln (1+x)$ for $i=1, \ldots, 8$. Also, $\tilde{\Lambda}_{i}(x)=\Lambda_{i}(x)=(2 x)^{0.5}$ for $i=9, \ldots, 15$. It follows that $\Gamma_{i}(\theta)=-\ln (1-\theta)$ for all $i=1, \ldots, 15$. We apply the procedure outlined in Section 6 to determine $\operatorname{good}\left(\theta_{u_{j}}: j \leq 10\right)$. As an illustration, consider the procedure to determine $\theta_{u_{1}}$. Here, we first need to minimize

$$
2 \ln \left(1+x_{1}\right)+2 \ln \left(1+x_{4}\right)+\sqrt{2 x_{11}}+\sqrt{2 x_{15}}
$$

subject to $x_{1}+x_{4}+x_{11}+x_{15} \geq u$ and $x_{i} \geq 0,1 \leq i \leq 4$. Note that $2 \ln (1+x)$ is dominated by $\sqrt{2 x}$ for all sufficiently large $x$. Due to the concavity of $\ln (1+x)$ it is easy to see that the minimum occurs around $\left(x_{1}, x_{4}, x_{11}, x_{15}\right)=(u, 0,0,0)$ or $(0, u, 0,0)$ (note that the solution also gives an indication of the most likely way $\left\{\mathcal{L}_{1}>u\right\}$ occurs: $X_{1}$ exceeds $u$ or $X_{4}$ exceeds $u$ ). The minimum value is $g(u)=2 \ln (1+u)$.

In the second step, we need to minimize

$$
\frac{1}{(1-\theta)^{8}} e^{-2 \theta g(u)}
$$

for $\theta \geq 0$. For $u$ sufficiently large, this yields

$$
\theta \equiv \theta_{u_{1}}=1-\frac{4}{g(u)} .
$$

Since $g(u)=\Lambda_{1}(u), \theta_{u_{1}}$ is of the form consistent with the requirement in Theorem 6. In general, since all $\mathcal{P}_{j}$ contain at least one Pareto distributed rv, it can be seen that

$$
\theta_{u_{j}}=1-\frac{n_{j}}{\tilde{\Lambda}_{1}(u)} .
$$

Case 2. Exponential-Tailed with Hazard Rate Twisting. In this case $X_{1}, \ldots, X_{8}$ have an exponential distribution with rate 0.5 and $X_{9}, \ldots, X_{15}$ have an exponential distribution with rate 1 . In this case $\alpha=1, \tilde{\lambda}_{i}=0.5$ for $i=1, \ldots, 8$, and $\tilde{\lambda}_{i}=1$ otherwise. Also, $\Gamma_{i}(\theta)=-\ln (1-\theta)$ for all $i=1, \ldots, 15$. The procedure in Section 6 yields

$$
\theta_{u_{j}}=1-\frac{n_{j}}{\lambda_{j}^{*} u}
$$

(recall that $\left.\lambda_{j}^{*}=\min _{i \in \mathcal{P}_{j}} \tilde{\lambda}_{i}\right)$.

Case 3. Superexponential-Tailed with Hazard Rate Twisting. In this case, $X_{1}, \ldots, X_{8}$ have the Weibull $(2,1)$ distribution and $X_{9}, \ldots, X_{15}$ have the Weibull $(2,2)$ distribution. Here $\alpha=2, \tilde{\lambda}_{i}=1$ for $i=1, \ldots, 8$, and $\tilde{\lambda}_{i}=4$ otherwise. Also, $\Gamma_{i}(\theta)=-\ln (1-\theta)$ for all $i=1, \ldots, 15$. The procedure in Section 6 yields

$$
\theta_{u_{j}}=1-\frac{n_{j}}{\lambda_{j}^{*} u^{\alpha}}
$$

where, recall that $\lambda_{j}^{*}$ is given by $(9)$. 
Case 4. Superexponential-Tailed with Asymptotic Hazard Rate Twisting. In this case $X_{1}, \ldots, X_{8}$ have the $N(1,0.02)$ distribution and $X_{9}, \ldots, X_{15}$ have the $N(1,0.01)$ distribution. In this case $\alpha=2$, and $\tilde{\lambda}_{i}=1 /\left(2 \sigma_{i}^{2}\right)$ for $i=1, \ldots, 15$ (where $\sigma_{i}^{2}$ denotes the variance of $X_{i}$ ). We twist each component $i$ using the asymptotic hazard function $\tilde{\Lambda}_{i}(x)=\left(x-\mu_{i}\right)^{2} /\left(2 \sigma_{i}^{2}\right)$. Hence $\Gamma_{i}(\theta)=-0.5 \ln (1-\theta)$ for all $i=1, \ldots, 15$. The procedure in Section 6 yields

$$
\theta_{u_{j}}=1-\frac{n_{j}}{2 \lambda_{j}^{*}\left(u-a_{j}\right)^{\alpha}}
$$

where $a_{j}$ is the mean of $\mathcal{L}_{j}$. As an illustration, consider $j=2$. Here, $n_{2}=3$, $a_{2}=\mu_{1}+\mu_{4}+\mu_{12}$ and $\lambda_{2}^{*}=\frac{1}{2\left(\sigma_{1}^{2}+\sigma_{4}^{2}+\sigma_{12}^{2}\right)}$. Then,

$$
\theta_{u_{2}}=1-\frac{3\left(\sigma_{1}^{2}+\sigma_{4}^{2}+\sigma_{12}^{2}\right)}{\left(u-\left(\mu_{1}+\mu_{4}+\mu_{12}\right)\right)^{2}} .
$$

Under asymptotic hazard rate twisting the distribution of each $X_{i}$ for $i=1,4$ and 12 , becomes $N\left(\mu_{i}, \sigma_{i}^{2} /\left(1-\theta_{u_{2}}\right)\right)$. In particular,

$$
\left(X_{1}+X_{4}+X_{12}\right) \sim N\left(\mu_{1}+\mu_{4}+\mu_{12}, \frac{\left(u-\left(\mu_{1}+\mu_{4}+\mu_{12}\right)\right)^{2}}{3}\right)
$$

under the new measure. Thus, the mean is unchanged but the variance is increased (so that the probability of exceeding $u$ is no longer rare).

Case 5. Superexponential-Tailed with Exponential Twisting. Same as Case 4, but now we apply the exponential-twisting based algorithm of Section 3.3 to each path. For each of the ten paths, we find the $\theta_{u_{j}}$ as the solution to (8). Note that $\mathcal{H}_{i}(\theta)=\mu_{i} \theta+\sigma_{i}^{2} \theta^{2} / 2$ for all $i=1, \ldots, 15$ (where $\mu_{i}$ and $\sigma_{i}^{2}$ denote the mean and variance of $X_{i}$ ). As an illustration, consider $j=2$. Here, the solution to (8) yields

$$
\theta_{u_{2}}=\left(u-\left(\mu_{1}+\mu_{4}+\mu_{12}\right)\right) /\left(\sigma_{1}^{2}+\sigma_{4}^{2}+\sigma_{12}^{2}\right) .
$$

Applying exponential twisting by amount $\theta_{u_{2}}$ to each $X_{i}$ in $\mathcal{P}_{2}$ makes $\left(X_{1}+X_{4}+X_{12}\right) \sim N\left(u, \sigma_{1}^{2}+\sigma_{4}^{2}+\sigma_{12}^{2}\right)$, that is, under the new measure, the mean duration of that path is changed to $u$ but the variance is unchanged.

For each case, we simulate for different values of $u$, and estimate the probability, the $99 \%$ relative error (i.e., the $99 \%$ confidence interval half-width upon the quantity to be estimated), the variance reduction factor (VRF) and the efficiency increase factor (EIF). The VRF is the ratio of the variance of the naive simulation estimator to that of the importance sampling estimator; the variance of the naive simulation estimator is estimated by substituting the accurate importance sampling estimator of $P(T>u)$ in $P(T>u)(1-P(T>u))$. The EIF is the ratio of the expected run-length of naive simulation to that of expected run-length of the importance sampling simulation, for achieving the same relative error. We estimate it by running naive simulation for the same number of replications as the importance sampling simulation, computing the ratio of the two CPU times, and then multiplying it with the VRF. We use MATLAB for the computation. Since MATLAB makes extensive use of matrix methods, 
Table I. Estimates of Probabilities, 99\% Relative Errors, Variance Reduction Factors (VRF), and Efficiency Increase Factors (EIF) for the Different Cases and for Various $u$ 's. The Paired Quantities in Brackets are (VRF, EIF)

\begin{tabular}{|c|c|c|c|c|c|}
\hline \multicolumn{2}{|c|}{ Case 1 (Subexp) } & \multicolumn{2}{|r|}{ Case $2(\operatorname{Exp})$} & \multicolumn{2}{|c|}{ Case 3 (Superexp) } \\
\hline$u$ & $\begin{array}{c}\text { Estimates } \\
(100,000 \text { rep })\end{array}$ & $u$ & $\begin{array}{c}\text { Estimates } \\
(100,000 \text { rep })\end{array}$ & $u$ & $\begin{array}{c}\text { Estimates } \\
(100,000 \text { rep })\end{array}$ \\
\hline 10 & $\begin{array}{c}2.41 * 10^{-1} \pm 1.1 \% \\
(1.7,0.7)\end{array}$ & 10 & $\begin{array}{c}.62 * 10^{-1} \pm .85 \% \\
(1.6, .55)\end{array}$ & 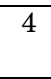 & $\begin{array}{c}1.36 * 10^{-1} \pm 1.2 \% \\
(2.7,1.2)\end{array}$ \\
\hline 20 & $\begin{array}{c}4.87 * 10^{-2} \pm 2.1 \% \\
(3.0,1.3)\end{array}$ & 15 & $\begin{array}{c}5.66 * 10^{-2} \pm 1.7 \% \\
(3.9,1.2)\end{array}$ & 5 & $\begin{array}{c}7.69 * 10^{-2} \pm 2.3 \% \\
(17,7.7)\end{array}$ \\
\hline 50 & $\begin{array}{c}4.18 * 10^{-3} \pm 4.6 \% \\
(7.4,3.1)\end{array}$ & 20 & $\begin{array}{c}6.80 * 10^{-3} \pm 2.5 \% \\
(15,5.2)\end{array}$ & 5.5 & $\begin{array}{c}1.21 * 10^{-3} \pm 2.9 \% \\
(65,32)\end{array}$ \\
\hline 100 & $\begin{array}{c}8.39 * 10^{-4} \pm 7.1 \% \\
(16,6.5)\end{array}$ & 25 & $\begin{array}{c}7.14 * 10^{-4} \pm 3.4 \% \\
(80,28)\end{array}$ & 6 & $\begin{array}{c}1.57 * 10^{-4} \pm 3.4 \% \\
(356,155)\end{array}$ \\
\hline 400 & $\begin{array}{c}4.70 * 10^{-5} \pm 11 \% \\
(109,45)\end{array}$ & 30 & $\begin{array}{c}7.39 * 10^{-5} \pm 4.2 \% \\
(498,142)\end{array}$ & 6.5 & $\begin{array}{c}1.61 * 10^{-5} \pm 4.0 \% \\
(2510,990)\end{array}$ \\
\hline 1600 & $\begin{array}{c}3.07 * 10^{-6} \pm 20 \% \\
(519,216)\end{array}$ & 35 & $\begin{array}{c}7.00 * 10^{-6} \pm 5.0 \% \\
(3708,1117)\end{array}$ & 7.0 & $\begin{array}{c}1.44 * 10^{-6} \pm 4.6 \% \\
(21444,9760)\end{array}$ \\
\hline
\end{tabular}

the CPU time is usually not proportional to the number of replications of the simulation. Hence, the EIF numbers (but not the VRF numbers) may change substantially if we change the number of replications.

Table I gives the results for the first three cases. Note that different sets of $u$ 's have been selected for the three cases, so that the estimated probabilities lie in similar ranges. The results are as expected; both the VRF and EIF become larger as $u$ increases.

Table II gives the results for Case 4 and Case 5, that apply different simulation algorithms to the same problem. Note that as $u$ increases, exponential twisting far exceeds asymptotic hazard rate twisting in terms of efficiency. Hence for the case of superexponential $X_{i}$ 's, whenever exponential twisting is easily implementable as in the Normal distribution case, we recommend using the exponential-twisting based algorithm of Section 3.3. In cases where exponential twisting is hard to implement (e.g., Case 3), asymptotic hazard rate twisting may be a viable alternative.

\subsection{Effect of Problem Size}

In this section, through simple examples we test the efficacy of proposed importance sampling techniques as $n$ increases. We consider two extreme cases for different values of $n$ :

(1) $\tau=1$. In PERT network terminology, this corresponds to all the activities in series.

(2) $\tau=n$. In PERT network terminology, this corresponds to all the activities in parallel.

We refer to the first as the series case and to the second as the parallel case. We observe that the variance reduction obtained by asymptotic hazard rate twisting deteriorates significantly with $n$ in the series case. The deterioration is insignificant in the parallel case. In contrast, exponential twisting, whenever applicable, is robust in both these dimensions. Recall that in the parallel case, 
Table II. Estimates of Probabilities, 99\% Relative Errors, Variance Reduction Factors (VRF), and Efficiency Increase Factors (EIF) for Normally Distributed Activity Durations, Using Two Different Methods. The Paired

Quantities in Brackets are (VRF, EIF)

\begin{tabular}{|c|c|c|}
\hline & Case 4 (Asymp. HRT) & Case 5 (Exp. Twist.) \\
\hline & Estimates & Estimates \\
$u$ & $(100,000 \mathrm{rep})$ & $(100,000$ rep $)$ \\
\hline \hline 4.3 & $2.57 * 10^{-1} \pm 1.1 \%$ & $2.61 * 10^{-1} \pm .71 \%$ \\
& $(1.5,1.3)$ & $(3.8,1.7)$ \\
\hline 4.5 & $5.61 * 10^{-2} \pm 2.7 \%$ & $5.61 * 10^{-2} \pm 0.97 \%$ \\
& $(1.6,1.4)$ & $(12,5.6)$ \\
\hline 4.7 & $6.31 * 10^{-3} \pm 5.0 \%$ & $6.20 * 10^{-3} \pm 1.17 \%$ \\
& $(4.2,3.3)$ & $(78,35)$ \\
\hline 4.9 & $3.60 * 10^{-4} \pm 9.3 \%$ & $3.57 * 10^{-4} \pm 1.3 \%$ \\
& $(21,18)$ & $(1030,471)$ \\
\hline 5.0 & $7.24 * 10^{-5} \pm 11 \%$ & $6.65 * 10^{-5} \pm 1.4 \%$ \\
& $(67,60)$ & $(4960,2350)$ \\
\hline 5.2 & $1.60 * 10^{-6} \pm 19 \%$ & $1.43 * 10^{-6} \pm 1.6 \%$ \\
& $(1137,890)$ & $(187610,89550)$ \\
\hline
\end{tabular}

Table III. Estimates of Variance Reduction Factors (VRF) Using Exponential Twisting and Asymptotic Hazard Rate Twisting, for Series and Parallel Cases. The Activity Durations are $N(0,1)$, and the $u$ is Selected so that $P(T>u)=0.0001$. (The VRF in the series column were analytically calculated.)

\begin{tabular}{|r|c|c|c|c|}
\hline & Series & Series & Parallel & Parallel \\
\hline \multicolumn{1}{|c|}{$n$} & VRF-exp & VRF-hazard & $\begin{array}{c}\text { VRF-exp } \\
(100,000 \text { rep })\end{array}$ & $\begin{array}{c}\text { VRF-hazard } \\
(100,000 \text { rep })\end{array}$ \\
\hline \hline 1 & 2388 & 317 & 2388 & 317 \\
\hline 5 & 2388 & 28 & 2085 & 296 \\
\hline 10 & 2388 & 5 & 2108 & 228 \\
\hline 50 & 2388 & 1 & 1980 & 190 \\
\hline 100 & 2388 & 1 & 1801 & 187 \\
\hline
\end{tabular}

both for exponential twisting and asymptotic hazard rate twisting, we use a mixture of appropriate probability distributions.

Specifically, we first consider the case where all the $X_{i}$ have the $N(0,1)$ distribution in the series and the parallel settings. We vary $n$, but at the same time vary $u$ so that $P(T>u)$ remains fixed at $10^{-4}$. Results with both aymptotic hazard rate twisting and exponential twisting are presented in Table III. In the series case, it is easy to compute the second moment in each setting analytically, so no simulations were conducted.

We also do the same for the case where all the $X_{i}$ 's have Pareto $(2,1)$ distribution. Results are presented in Table IV. Here, in each setting $u$ was determined through trial-and-error so that $P(T>u)$ is approximately $10^{-4}$.

\section{CONCLUSION AND FUTURE WORK}

In this article, we developed tail asymptotics and fast simulation techniques for estimating the probability that the maximum of sums of a few random variables 
Table IV. Estimates of Variance

Reduction Factors (VRF) Using Asymptotic Hazard Rate Twisting, for Series and Parallel Cases. The Activity Durations are Pareto(2,1), and the $u$ is Selected so that $P(T>u)=0.0001$

\begin{tabular}{|r|c|c|}
\hline & Series & Parallel \\
\hline$n$ & $\begin{array}{c}\text { VRF-Hazard } \\
(100,000 \text { rep })\end{array}$ & $\begin{array}{c}\text { VRF-Hazard } \\
(100,000 \text { rep })\end{array}$ \\
\hline \hline 1 & 875 & 875 \\
\hline 5 & 68 & 673 \\
\hline 10 & 14 & 633 \\
\hline 50 & 1 & 575 \\
\hline 100 & 1 & 540 \\
\hline
\end{tabular}

exceeds a large threshold. We considered both light and heavy-tailed random variables and introduced asymptotic hazard rate twisting based importance sampling in these settings. This may have implementation advantages over hazard rate twisting or exponential twisting, although the latter whenever easy to implement, is empirically seen to perform much better. We also noted that straightforward application of exponential twisting and asymptotic hazard rate twisting may not be very effective in estimating $P(T>u)$ when the associated PERT network has many paths. This may be remedied by using a probability measure that is a mixture of probability measures so that for each path there is a probability measure in this mixture that is tailored to it.

Our work focussed on small number of random variables. As mentioned in the introduction, one useful generalization corresponds to extending these results to large networks when the random variables involved are light tailed. Then the tail asymptotics may be developed with the help of large deviations theory. In addition, it may be straightforward to extend the exponential twisting based importance sampling methods discussed here to large networks.

Consider a resource allocation problem where the distribution time of each activity is a function of the resources allocated to it. Further suppose that the objective function corresponds to the probability of large delays. In this setting, the tail asymptotics developed here may serve as a surrogate objective function and facilitate determining the asymptotic solution to this resource allocation problem. The authors explore this in future research.

APPENDIX: PROOFS

Proofs for Section 3: Light Tails, Exponential Twisting

We first prove some lemmas useful in proving Theorem 3.2.

Lemma 8.1. Under Assumption 3.1,

$$
\liminf _{u \rightarrow \infty} \frac{\log P\left(S_{n}>u\right)}{u^{\alpha}} \geq-\lambda^{*} .
$$


Proof. First consider the case where $\alpha=1$. Since all $X_{i}$ 's are real valued random variables, there exist constants $\left(b_{i}: 2 \leq i \leq n\right)$ such that for each such $i, P\left(X_{i} \geq b_{i}\right)>0$. Thus,

$$
P\left(S_{n}>u\right) \geq P\left(X_{1} \geq u-\sum_{i=2}^{n} b_{i}\right) \prod_{i=2}^{n} P\left(X_{i} \geq b_{i}\right) .
$$

From this, it easily follows that

$$
\liminf _{u \rightarrow \infty} \frac{\log P\left(S_{n}>u\right)}{u} \geq-\tilde{\lambda}_{1} .
$$

Similarly, the above inequality holds for right-hand side equal to $-\tilde{\lambda}_{i}$ for $2 \leq$ $i \leq k$ and hence for $-\lambda^{*}=-\min _{i \leq k} \tilde{\lambda}_{i}$.

Now consider $\alpha>1$. Suppose that $\left(\beta_{i} \geq 0: i \leq k\right)$ are such that $\sum_{i \leq k} \beta_{i}=1$. Again,

$$
P\left(S_{n}>u\right) \geq\left[\prod_{i \leq k} P\left(X_{i} \geq \beta_{i}\left(u-\sum_{j>k}^{n} b_{j}\right)\right)\right]\left[\prod_{j>k}^{n} P\left(X_{j} \geq b_{j}\right)\right],
$$

(with the usual convention that if $k=n$, then $\sum_{j>k}^{n} b_{j}=0$ and $\Pi_{j>k}^{n} P\left(X_{j} \geq\right.$ $\left.b_{j}\right)=1$ ). Let $\bar{b}=\sum_{j>k}^{n} b_{j}$. Thus

$$
\frac{\log P\left(S_{n}>u\right)}{u^{\alpha}} \geq \sum_{i \leq k} \frac{\log P\left(X_{i} \geq \beta_{i}(u-\bar{b})\right)}{u^{\alpha}}+\sum_{j>k} \frac{\log P\left(X_{j} \geq b_{j}\right)}{u^{\alpha}} .
$$

Taking lim inf as $u \rightarrow \infty$, from Assumption 3.1, it follows that

$$
\liminf _{u \rightarrow \infty} \frac{\log P\left(S_{n}>u\right)}{u^{\alpha}} \geq-\sum_{i \leq k} \beta_{i}^{\alpha} \lambda_{i}
$$

Now by setting $\beta_{i}=1 / \tilde{\lambda}_{i}^{1 /(\alpha-1)} / \sum_{j \leq k} 1 / \tilde{\lambda}_{j}^{1 /(\alpha-1)}$ the required relation (33) follows.

To prove Theorem 3.2 for $\alpha>1$ case, we need to develop an asymptotic upper bound for log moment generating function in this setting. To this end, we begin with a simple observation regarding the log-moment generating function. Let $\mathcal{H}$ be the log-moment generating function corresponding to a distribution function $F$. Using $\exp [\theta s]=1+\theta \int_{0}^{s} \exp [\theta x] d x$, we get

$$
\begin{aligned}
\int_{0}^{\infty} \exp [\theta s] d F(s) & =\bar{F}(0)+\theta \int_{0}^{\infty} \int_{0}^{s} \exp [\theta x] d x d F(s) \\
& =\bar{F}(0)+\theta \int_{0}^{\infty} \exp [\theta x] \bar{F}(x) d x
\end{aligned}
$$

Since, for $\theta>0, \int_{-\infty}^{0} \exp [\theta s] d F(s) \leq F(0)$, it follows that

$$
\exp [\mathcal{H}(\theta)] \leq 1+\theta \int_{0}^{\infty} \exp [\theta x] \bar{F}(x) d x \quad \forall \theta>0 .
$$

Let $\log ^{+}(\theta)$ denote $\max (\log \theta, 1)$. 
Lemma 8.2. Suppose that the tail distribution function $\bar{F}($.$) of a random$ variable satisfies the asymptotic relation

$$
\limsup _{u \rightarrow \infty} \frac{\log \bar{F}(u)}{u^{\alpha}} \leq-\lambda, \quad 0<\lambda<\infty
$$

for $\alpha>1$ and $\lambda>0$. Then for every sufficiently small $\epsilon$ there exists a constant $C_{\epsilon}$ and constants $C_{1}$ and $C_{2}$ that are independent of $\theta$ such that

$$
\mathcal{H}(\theta) \leq\left(\frac{(\theta / \alpha)^{\alpha /(\alpha-1)}}{(\lambda-\epsilon)^{1 /(\alpha-1)}}\right)(\alpha-1)+C_{\epsilon}+C_{1} \log ^{+}(\theta)+C_{2} \log ^{+}(\lambda-\epsilon) .
$$

Proof. Given $\epsilon>0$, it follows from (35) that there exists $K_{\epsilon}$ such that

$$
\bar{F}(x) \leq K_{\epsilon} \exp \left[-(\lambda-\epsilon) x^{\alpha}\right],
$$

for all $x \geq 0$. Using (34) and Lemma 8.3 given below, it follows that

$$
\begin{aligned}
\mathcal{H}(\theta) & \leq 1+\log ^{+}(\theta)+\log ^{+}\left(\int_{0}^{\infty} \exp [\theta x] \bar{F}(x) d x\right), \\
& \leq 1+\log ^{+}(\theta)+\log ^{+}\left(K_{\epsilon} \int_{0}^{\infty} \exp \left[\theta x-(\lambda-\epsilon) x^{\alpha}\right] d x\right) .
\end{aligned}
$$

This in turn is upper bounded by

$$
1+\log ^{+}(\theta)+\log ^{+} K_{\epsilon}+\left(\frac{(\theta / \alpha)^{\alpha /(\alpha-1)}}{(\lambda-\epsilon)^{1 /(\alpha-1)}}\right)(\alpha-1)+K_{1} \log ^{+}(\theta)+K_{2} \log ^{+}(\lambda-\epsilon) .
$$

The result follows from this.

Lemma 8.3. For $\alpha>1$ and $\gamma>0$, there exist constants $K_{1}$ and $K_{2}$ independent of $\theta>0$ such that

$$
\log \left(\int_{0}^{\infty} \exp \left[\theta x-\gamma x^{\alpha}\right] d x\right) \leq\left(\frac{(\theta / \alpha)^{\alpha /(\alpha-1)}}{\gamma^{1 /(\alpha-1)}}\right)(\alpha-1)+K_{1} \log ^{+}(\theta)+K_{2} \log ^{+}(\gamma) .
$$

Proof. Consider the integral

$$
\int_{0}^{\infty} \exp \left[\theta x-\gamma x^{\alpha}\right] d x
$$

Let $\tilde{x}=\left(\frac{\theta}{\gamma \alpha}\right)^{1 /(\alpha-1)}$ so that $\theta=\gamma \alpha \tilde{x}^{\alpha-1}$. From Taylor's expansion, it follows that

$$
x^{\alpha}=\tilde{x}^{\alpha}+(x-\tilde{x}) \alpha \tilde{x}^{\alpha-1}+\frac{(x-\tilde{x})^{2}}{2} \alpha(\alpha-1) \xi_{x}^{\alpha-2},
$$

where $\xi_{x}$ lies between $\tilde{x}$ and $x$. Thus, (37) may be re-expressed as

$$
\exp \left[\theta \tilde{x}-\gamma \tilde{x}^{\alpha}\right] \int_{0}^{\infty} \exp \left[-\frac{(x-\tilde{x})^{2}}{2} \gamma \alpha(\alpha-1) \xi_{x}^{\alpha-2}\right] d x
$$

Note that

$$
\theta \tilde{x}-\gamma \tilde{x}^{\alpha}=\left(\frac{(\theta / \alpha)^{\alpha /(\alpha-1)}}{\gamma^{1 /(\alpha-1)}}\right)(\alpha-1) .
$$

ACM Transactions on Modeling and Computer Simulation, Vol. 17, No. 2, Article 7, Publication date: April 2007. 
Thus, to complete the proof, it remains to prove that

$$
\log \left(\int_{0}^{\infty} \exp \left[-\frac{(x-\tilde{x})^{2}}{2} \gamma \alpha(\alpha-1) \xi_{x}^{\alpha-2}\right] d x\right) \leq K_{1} \log ^{+}(\theta)+K_{2} \log ^{+}(\gamma)
$$

for constants $K_{1}$ and $K_{2}$ independent of $\theta$. Now,

$$
\begin{array}{r}
\int_{0}^{\infty} \exp \left[-\frac{(x-\tilde{x})^{2}}{2} \gamma \alpha(\alpha-1) \xi_{x}^{\alpha-2}\right] d x \leq 2 \tilde{x} \\
+\int_{2 \tilde{x}}^{\infty} \exp \left[-\frac{(x-\tilde{x})^{2}}{2} \gamma \alpha(\alpha-1) \xi_{x}^{\alpha-2}\right] d x .
\end{array}
$$

Consider the case $\alpha \geq 2$, so that $\xi_{x}^{\alpha-2} \geq \tilde{x}^{\alpha-2}$ for $x \geq \tilde{x}$. Hence, the integral in the right-hand side of (40) is bounded by

$$
\int_{-\infty}^{\infty} \exp \left[-\frac{(x-\tilde{x})^{2}}{2} \gamma \alpha(\alpha-1) \tilde{x}^{\alpha-2}\right] d x
$$

This integral equals $\frac{\sqrt{2 \pi}}{\sqrt{\gamma \alpha(\alpha-1) \tilde{x}^{\alpha-2}}}$ and hence (39) is true.

Now consider the case where $\alpha<2$. Here, $\xi_{x}^{\alpha-2} \geq x^{\alpha-2}$ for $x \geq \tilde{x}$ and $(x-\tilde{x})^{2} \geq$ $(x / 2)^{2}$ for $x \geq 2 \tilde{x}$ and hence the integral in the right-hand side of (40) is bounded by

$$
\int_{0}^{\infty} \exp \left[-\frac{x^{\alpha}}{8} \gamma \alpha(\alpha-1)\right] d x=\gamma^{\frac{-1}{\alpha}} \int_{0}^{\infty} \exp \left[-\frac{y^{\alpha}}{8} \alpha(\alpha-1)\right] d y .
$$

Thus, (39) is true.

Proof of Theorem 3.2. First consider $\alpha>1$. Fix $\epsilon>0$. Recall that $\log ^{+} x$ denotes $\max (\log x, 1)$. From Lemma 8.2 and the fact that $\theta_{u} \sim \alpha \lambda^{*} u^{\alpha-1}$, it follows that for $i \leq k$ and $u$ sufficiently large, $\mathcal{H}_{i}\left(\theta_{u}\right)$ is $\leq$

$\lambda^{* \frac{\alpha}{\alpha-1}} u^{\alpha} \frac{1}{\left(\tilde{\lambda}_{i}-\epsilon\right)^{1 /(\alpha-1)}}(\alpha-1)+o\left(u^{\alpha}\right)+C_{\epsilon}+C_{1} \log ^{+}\left(\alpha \lambda^{*} u^{\alpha-1}\right)+C_{2} \log ^{+}\left(\tilde{\lambda}_{i}-\epsilon\right)$

and for $i>k$, for any $M<\infty, \mathcal{H}_{i}\left(\theta_{u}\right)$ is $\leq$

$\lambda^{* \frac{\alpha}{\alpha-1}} u^{\alpha} \frac{1}{(M-\epsilon)^{1 /(\alpha-1)}}(\alpha-1)+o\left(u^{\alpha}\right)+C_{\epsilon}+C_{1} \log ^{+}\left(\alpha \lambda^{*} u^{\alpha-1}\right)+C_{2} \log ^{+}(M-\epsilon)$.

It is easy to select $C_{\epsilon}, C_{1}$ and $C_{2}$ so that they are independent of $i$ and $u$. Recall that for $u$ sufficiently large so that $\theta_{u}>0$,

$$
L_{\theta_{u}}=\exp \left[-\theta_{u} S_{n}+\sum_{i \leq n} \mathcal{H}_{i}\left(\theta_{u}\right)\right] \leq \exp \left[-\theta_{u} u+\sum_{i \leq n} \mathcal{H}_{i}\left(\theta_{u}\right)\right] \text { a.s. }
$$

It then follows that

$$
P\left(S_{n}>u\right)=E_{P_{\theta_{u}}} L_{\theta_{u}} I\left(S_{n}>u\right) \leq \exp \left[-\theta_{u} u+\sum_{i \leq n} \mathcal{H}_{i}\left(\theta_{u}\right)\right] .
$$

Using the estimates in (41) and (42), recalling that $\theta_{u} \sim \alpha \lambda^{*} u^{\alpha-1}$, taking the logarithms, dividing by $u^{\alpha}$ and taking limsup, we get $\lim \sup _{u \rightarrow \infty} \frac{\log P\left(S_{n}>u\right)}{u^{\alpha}}$ is 
less than or equal to

$$
-\alpha \lambda^{*}+\sum_{i \leq k} \lambda^{* \frac{\alpha}{\alpha-1}} \frac{1}{\left(\tilde{\lambda}_{i}-\epsilon\right)^{1 /(\alpha-1)}}(\alpha-1)+\sum_{i>k} \lambda^{* \frac{\alpha}{\alpha-1}} \frac{1}{(M-\epsilon)^{1 /(\alpha-1)}}(\alpha-1) .
$$

This holds for all sufficiently small $\epsilon>0$ and $M<\infty$. Thus, taking limit as $M \rightarrow \infty$ and then limit as $\epsilon \rightarrow 0$, it follows that

$$
\limsup _{u \rightarrow \infty} \frac{\log P\left(S_{n}>u\right)}{u^{\alpha}} \leq-\alpha \lambda^{*}+\sum_{i \leq k} \lambda^{* \frac{\alpha}{\alpha-1}} \frac{1}{\tilde{\lambda}_{i}^{1 /(\alpha-1)}}(\alpha-1) .
$$

The right-hand side above equals $-\lambda^{*}$ and (6) follows from this and Lemma 8.1. Similarly, by essentially repeating the analysis from (43) onwards, $E_{P_{\theta_{u}}}\left[L_{\theta_{u}}^{2} I\left(S_{n}>u\right)\right]$ may be bounded from above to establish (7).

Now consider $\alpha=1$. Without loss of generality, let $\lambda_{1}=\min _{i \leq k} \tilde{\lambda}_{i}$. Again,

$$
P\left(S_{n}>u\right)=E_{P_{\theta_{u}}}\left[L_{\theta_{u}} I\left(S_{n}>u\right)\right] \leq \exp \left[-\theta_{u} u+\sum_{i \leq n} \mathcal{H}_{i}\left(\theta_{u}\right)\right],
$$

and

$$
E_{P_{\theta}}\left[L_{\theta_{u}}^{2} I\left(S_{n}>u\right)\right] \leq \exp \left[-2 \theta_{u} u+2 \sum_{i \leq n} \mathcal{H}_{i}\left(\theta_{u}\right)\right] .
$$

Since $\theta_{u} \sim \lambda^{*}$ and each $\mathcal{H}_{i}\left(\theta_{u}\right)=o(u)$, the result follows.

Proof of Theorem 3.5. From Theorem 3.2, it follows that when $\tilde{\mathcal{P}}_{j}$ is nonempty, then

$$
\lim _{u \rightarrow \infty} \frac{\log P\left(\mathcal{L}_{j}>u\right)}{u^{\alpha}}=-\lambda_{j}^{*} .
$$

Otherwise, if $\tilde{\mathcal{P}}_{j}$ is empty, then

$$
P\left(\mathcal{L}_{j}>u\right) \leq P\left(\bigcup_{i \in \mathcal{P}_{j}}\left\{X_{i}>u /\left|\mathcal{L}_{j}\right|\right\}\right) \leq \sum_{i \in \mathcal{P}_{j}} P\left(X_{i}>u /\left|\mathcal{L}_{j}\right|\right) .
$$

Since, due to Assumption 3.1, $\frac{\Lambda_{i}\left(u /\left|\mathcal{L}_{j}\right|\right)}{u^{\alpha}} \rightarrow \infty$, it follows that

$$
\lim _{u \rightarrow \infty} \frac{1}{u^{\alpha}} \log P\left(\mathcal{L}_{j}>u\right)=-\infty .
$$

The proof now follows from the inequalities

$$
\begin{aligned}
\max _{j \leq \tau} P\left(\mathcal{L}_{j}>u\right) & \leq P(T>u)=P\left(\bigcup_{j \leq \tau} \mathcal{L}_{j}>u\right) \\
& \leq \sum_{j \leq \tau} P\left(\mathcal{L}_{j}>u\right) \\
& \leq \tau \max _{j \leq \tau} P\left(\mathcal{L}_{j}>u\right) .
\end{aligned}
$$

Taking logarithms in the above inequalities, dividing by $u^{\alpha}$, and taking limits as $u \rightarrow \infty$, the result follows. 
Proof of Theorem 3.8. Note again that

$$
E_{P^{*}}\left[L^{2} I(T>u)\right] \leq \sum_{j \in K} E_{P^{*}}\left[L^{2} I\left(\mathcal{L}_{j}>u\right)\right] .
$$

Thus, it suffices to show that

$$
\limsup _{u \rightarrow \infty} \frac{\log E_{P^{*}}\left[L^{2} I\left(\mathcal{L}_{j}>u\right)\right]}{u^{\alpha}} \leq-2 \lambda_{j}^{*},
$$

for all $j \leq \tau$ (recall that $\lambda_{j}^{*}=\infty$ if $\tilde{\mathcal{P}}_{j}$ is empty). To see this, first consider $j$ such that $\tilde{\mathcal{P}}_{j}$ is nonempty. Again consider the expression for $L$ given in (14). This may be upper bounded by

$$
\frac{1}{p_{j}} \exp \left[-\theta_{u_{j}} \sum_{i \in \mathcal{P}_{j}} X_{i}+\sum_{i \in \mathcal{P}_{j}} \mathcal{H}_{i}\left(\theta_{u_{j}}\right)\right] .
$$

Thus, (45) follows from the arguments used in proof of Theorem 3.2 specialized to activities in $\mathcal{P}_{j}$.

Now consider the case $j$ such that $\tilde{\mathcal{P}}_{j}$ is empty. Note that $E_{P^{*}}\left[L^{2} I\left(\mathcal{L}_{j}>u\right)\right]=$ $E_{P}\left[L I\left(\mathcal{L}_{j}>u\right)\right]$ and that $L$ is uniformly upper bounded by $\frac{1}{p_{j}}$ along the sample paths in the set $\left\{\mathcal{L}_{j}>u\right\}$. Thus,

$$
E_{P}\left[L I\left(\mathcal{L}_{j}>u\right)\right] \leq \frac{1}{p_{j}} P\left(\mathcal{L}_{j}>u\right) .
$$

The result then follows from (44).

Proofs for Section 4: Light Tails, Hazard Rate Twisting

The following lemma is useful in proving Theorem 4.1.

Lemma 8.4. If $\tilde{\Lambda}_{i}$ satisfies (18), then

$$
\exp \left[\Gamma_{i}(\theta)\right] \leq p\left(\frac{1}{1-\theta}\right)
$$

for $0<\theta<1$, where $p(x)$ is a polynomial in $x$.

Proof. We need to show that

$$
\int_{-\infty}^{\infty} \exp \left[\theta \tilde{\Lambda}_{i}(x)\right] \lambda_{i}(x) \exp \left(-\Lambda_{i}(x)\right) d x
$$

is upper bounded by a polynomial term in $\frac{1}{1-\theta}$. From (18), we can upper bound this term by

$$
\int_{-\infty}^{\infty} \lambda_{i}(x) \exp \left[a_{i} \max \left(\log \Lambda_{i}(x), 1\right)-(1-\theta) \Lambda_{i}(x)\right] d x
$$

Now simply set $y=(1-\theta) \Lambda_{i}(x)$, then $d y=(1-\theta) \lambda_{i}(x) d x$. Note that $\Lambda_{i}(-\infty)=0$ and $\Lambda_{i}(\infty)=\infty$. Thus, the above integral is upper bounded by

$$
\frac{1}{(1-\theta)^{a_{i}+1}} \int_{0}^{\infty} \max (\exp [1], y)^{a_{i}} \exp [-y] d y,
$$

establishing the result.

ACM Transactions on Modeling and Computer Simulation, Vol. 17, No. 2, Article 7, Publication date: April 2007. 
Proof of Theorem 4.1. We first establish (21). In view of Lemma 8.1, it suffices to show that

$$
\limsup _{u \rightarrow \infty} \frac{\log E_{P^{*}}\left[L^{2} I\left(S_{n}>u\right)\right]}{u^{\alpha}} \leq-2 \lambda^{*} .
$$

Some notation is useful in displaying intermediate results used to prove (21). For $M>0$, let

$$
A_{M}=\left\{S_{n}>u\right\} \cap \bigcap_{i>k}\left\{X_{i} \leq u / M\right\}
$$

and

$$
B_{M}=\bigcup_{i>k}\left\{X_{i}>u / M\right\} .
$$

Note that $\left\{S_{n}>u\right\} \subset A_{M} \cup B_{M}$. Equation (21) follows easily from Lemmas 8.5 and 8.6 stated below.

To complete the proof of Theorem 4.1 note that

$$
E_{P^{*}}\left[L^{2} I(T>u)\right] \leq \sum_{j \leq \tau} E_{P^{*}}\left[L^{2} I\left(\mathcal{L}_{j}>u\right)\right] .
$$

Thus, it suffices to show that

$$
\limsup _{u \rightarrow \infty} \frac{\log E_{P^{*}}\left[L^{2} I\left(\mathcal{L}_{j}>u\right)\right]}{u^{\alpha}} \leq-2 \lambda_{j}^{*} .
$$

To see this, first consider $j$ such that $\tilde{\mathcal{P}}_{j}$ is nonempty. Consider again the expression for $L$ given in (19). This may be bounded from above by

$$
\exp \left[-\theta_{u} \sum_{i \in \tilde{\mathcal{P}}_{j}} \tilde{\Lambda}_{i}\left(X_{i}\right)+\sum_{i \in \tilde{\mathcal{P}}_{j}} \Gamma_{i}\left(\theta_{u}\right)+o\left(u^{\alpha}\right)\right] \text { a.s. }
$$

where we use the fact that $\sum_{i \leq k, i \notin \tilde{\mathcal{P}}_{j}} \Gamma_{i}\left(\theta_{u}\right)$ is $\exp \left[o\left(u^{\alpha}\right)\right]$ (see Lemma 8.4). The proof follows as in the proof of $(21)$, with the caveat that the additional $\exp \left[o\left(u^{\alpha}\right)\right]$ in the likelihood ratio in no way affects that proof.

Now consider the case $j$ such that $\tilde{\mathcal{P}}_{j}$ is empty. Note that $E_{P^{*}}\left[L^{2} I\left(\mathcal{L}_{j}>\right.\right.$ $u)]=E_{P}\left[L I\left(\mathcal{L}_{j}>u\right)\right]$. Again, note that $L$ is uniformly upper bounded by an $\exp \left[o\left(u^{\alpha}\right)\right]$ term on the set $\left\{\mathcal{L}_{j}>u\right\}$. Thus,

$$
E_{P}\left[L I\left(\mathcal{L}_{j}>u\right)\right] \leq \exp \left[o\left(u^{\alpha}\right)\right] P\left(\mathcal{L}_{j}>u\right) .
$$

The result then follows from (44).

Lemma 8.5. Under Assumption 3.1, (17) and (18),

$$
\lim _{M \rightarrow \infty} \limsup _{u \rightarrow \infty} \frac{\log E_{P^{*}}\left[L^{2} I\left(A_{M}\right)\right]}{u^{\alpha}} \leq-2 \lambda^{*}
$$

Lemma 8.6. Under Assumption 3.1, (17) and (18),

$$
\lim _{M \rightarrow \infty} \limsup _{u \rightarrow \infty} \frac{\log E_{P^{*}}\left[L^{2} I\left(B_{M}\right)\right]}{u^{\alpha}} \leq-2 \lambda^{*}
$$


Proof of Lemma 8.5. Due to Lemma 8.4, $(1-\theta) \Gamma_{i}(\theta) \rightarrow 0$ as $\theta \uparrow 1$, and hence,

$$
\frac{\Gamma_{i}\left(\theta_{u}\right)}{\Theta\left(u^{\alpha}\right)} \rightarrow 0
$$

as $u \rightarrow \infty$. In the following discussion we assume that $u$ is sufficiently large so that $0<\theta_{u}<1$. Recall display (19) and note that $A_{M} \subset\left\{\sum_{i=1}^{k} X_{i}>u\left(1-\frac{n-k}{M}\right)\right\}$. In view of these, it suffices to show that for any positive $\epsilon<1$, there exists a constant $\tilde{C}_{\epsilon}$ such that,

$$
\begin{aligned}
\exp \left[-\theta_{u} \sum_{i=1}^{k} \tilde{\Lambda}_{i}\left(X_{i}\right)\right] I\left(\sum_{i=1}^{k} X_{i}\right. & \left.>u\left(1-\frac{n-k}{M}\right)\right) \\
& \leq \exp \left[-\lambda^{*}(1-\epsilon) \theta_{u} u^{\alpha}\left(1-\frac{n-k}{M}\right)^{\alpha}+\tilde{C}_{\epsilon}\right]
\end{aligned}
$$

as such. The result then follows by squaring the above equation, taking expectation on both sides with respect to $P^{*}$ (note that the right-hand side is deterministic), dividing the logarithm of the resultant value of by $u^{\alpha}$, taking the limit as $u \rightarrow \infty$, and letting $\epsilon \rightarrow 0$ and $M \rightarrow \infty$ in the right-hand side.

To see (51), note that since $\tilde{\Lambda}_{i}$ is non-negative and $\tilde{\Lambda}_{i}(x) \sim \tilde{\lambda}_{i} x^{\alpha}$, it follows that for $x \geq 0$, for any positive $\epsilon<1$, there exists a positive constant $C_{\epsilon}$ such that

$$
\tilde{\Lambda}_{i}(x) \geq \tilde{\lambda}_{i} x^{\alpha}(1-\epsilon)-C_{\epsilon} .
$$

Using this, and letting $\mathcal{I}$ denote the random set of rvs amongst $\left(X_{1}, \ldots, X_{k}\right)$ that are positive, we can upper bound the left-hand side of (51) with

$$
\exp \left[-\theta_{u}(1-\epsilon) \sum_{i \in \mathcal{I}} \tilde{\lambda}_{i} X_{i}^{\alpha}+k C_{\epsilon}\right] I\left(\sum_{i=1}^{k} X_{i}>u\left(1-\frac{n-k}{M}\right)\right) .
$$

Using Jensen's inequality, it is easy to infer that

$$
\sum_{i \in \mathcal{I}} \tilde{\lambda}_{i} x_{i}^{\alpha} \geq \frac{1}{\left(\sum_{i \in \mathcal{I}} 1 / \tilde{\lambda}_{i}^{\frac{1}{\alpha-1}}\right)^{\alpha-1}}\left(\sum_{i \in \mathcal{I}} x_{i}\right)^{\alpha}
$$

To see this, consider a rv that takes value $\lambda_{j}^{\frac{1}{\alpha-1}} x_{j}$ with probability $1 / \lambda_{j}^{\frac{1}{\alpha-1}} / \sum_{i \in \mathcal{I}} 1 / \tilde{\lambda}_{i}^{\frac{1}{\alpha-1}}$ for $j \in \mathcal{I}$, and use the fact that $\alpha$ moment of a non-negative $\mathrm{rv}$ is greater than or equal to the first moment raised to power $\alpha$ for $\alpha \geq 1$. Note that

$$
\frac{1}{\left(\sum_{i \in \mathcal{I}} 1 / \tilde{\lambda}_{i}^{\frac{1}{\alpha-1}}\right)^{\alpha-1}} \geq \lambda^{*}
$$

Thus, the left-hand side of (51) may be upper bounded by

$$
\exp \left[-\theta_{u}(1-\epsilon) \lambda^{*}\left(\sum_{i=1}^{k} X_{i}\right)^{\alpha}+k C_{\epsilon}\right] I\left(\sum_{i=1}^{k} X_{i}>u\left(1-\frac{n-k}{M}\right)\right),
$$

and (51) follows. 
Proof of Lemma 8.6. Note that $E_{P^{*}}\left[L^{2} I\left(B_{M}\right)\right]=E_{P}\left[L I\left(B_{M}\right)\right]$. From (50), and the fact that $\tilde{\Lambda}(x) \geq 0$ for all $x$ in (19), it follows that $L \leq \exp \left[o\left(u^{\alpha}\right)\right]$ as such. Thus, $E_{P}\left[L I\left(B_{M}\right)\right] \leq \exp \left[o\left(u^{\alpha}\right)\right] P\left(B_{M}\right)$. It thus suffices to show that

$$
\lim _{M \rightarrow \infty} \limsup _{u \rightarrow \infty} \frac{\log P\left(B_{M}\right)}{u^{\alpha}} \leq-2 \lambda^{*}
$$

Note that

$$
P\left(\bigcup_{i>k} X_{i}>u / M\right) \leq \sum_{i>k} P\left(X_{i}>u / M\right)
$$

and $P\left(X_{i}>u / M\right)=\exp \left(-\Lambda_{i}(u / M)\right)$. Hence, it suffices to show that for $i>k$

$$
\lim _{M \rightarrow \infty} \liminf _{u \rightarrow \infty} \frac{\Lambda_{i}(u / M)}{u^{\alpha}} \geq 2 \lambda^{*} .
$$

From Assumption 3.1, for any $M, \frac{\Lambda_{i}(u / M)}{\left(\frac{u}{M}\right)^{\alpha}}$ increases to infinity. Hence, $\liminf _{u \rightarrow \infty} \frac{\Lambda_{i}(u / M)}{u^{\alpha}} \geq 2 \lambda^{*}$ and the result follows.

Proofs for Section 5: Heavy Tails

Proof of Theorem 5.2. Note that,

$$
\begin{aligned}
P(T>u) & \geq P\left(\bigcup_{i \leq n}\left\{X_{i}>u\right\}\right) \\
& \geq \sum_{i \leq n} P\left(X_{i}>u\right)-\sum_{i, j, i \neq j} P\left(X_{i}>u\right) P\left(X_{j}>u\right) .
\end{aligned}
$$

Hence, dividing both sides by $P\left(X_{1}>u\right)$, it is easily seen that

$$
\liminf _{u \rightarrow \infty} \frac{P(T>u)}{P\left(X_{1}>u\right)} \geq \sum_{i=1}^{k} \gamma_{i}
$$

Also note that,

$$
P(T>u) \leq P\left(S_{n}>u\right),
$$

so from (22), it follows that

$$
\limsup _{u \rightarrow \infty} \frac{P(T>u)}{P\left(X_{1}>u\right)} \leq \sum_{i=1}^{k} \gamma_{i} .
$$

Proof of Theorem 5.4. Note that the likelihood ratio of $P$ with respect to $P^{*}$ equals

$$
L=\exp \left[-\theta_{u} \sum_{i=1}^{n} \tilde{\Lambda}_{i}\left(X_{i}\right)+\sum_{i=1}^{n} \Gamma_{i}\left(\theta_{u}\right)\right] .
$$

In view of (26) and the fact that each $\tilde{\Lambda}_{i}$ is non-negative, it follows that there exists a constant $D_{\epsilon}>0$ such that

$$
(1-\epsilon) \tilde{\Lambda}_{1}(x)-D_{\epsilon} \leq \tilde{\Lambda}_{i}(x),
$$


for all $i$ and $x$. Using this, for $u$ sufficiently large, $L$ may be upper bounded by

$$
\exp \left[n D_{\epsilon}-\theta_{u}(1-\epsilon) \sum_{i=1}^{n} \tilde{\Lambda}_{1}\left(X_{i}\right)+\sum_{i=1}^{n} \Gamma_{i}\left(\theta_{u}\right)\right] .
$$

In particular, for $u \geq u_{\epsilon}$, using (27), noting that $\tilde{\Lambda}_{1}\left(S_{n}\right) \geq \tilde{\Lambda}_{1}(u)$ when $S_{n}>u$ for $u$ sufficiently large, it follows that

$L^{2} I(T>u) \leq L^{2} I\left(S_{n}>u\right) \leq \exp \left[2 n D_{\epsilon}-2 \theta_{u}(1-\epsilon) \tilde{\Lambda}_{1}(u)+2 \theta_{u} \epsilon(1-\epsilon)+2 \sum_{i=1}^{n} \Gamma_{i}\left(\theta_{u}\right)\right]$.

In particular, the logarithm of the right-hand side upper bounds $\log E_{P^{*}}\left[L^{2} I(T>u)\right]$. The result now follows by noting that due to (25) and Lemma $8.4, \Gamma_{i}\left(\theta_{u}\right)$ is bounded from above by $\log p\left[\Theta\left(\tilde{\Lambda}_{1}(u)\right)\right]\left(\right.$ since $\tilde{\Lambda}_{1}(u)=$ $\left.1 /\left(1-\theta_{u}\right)\right)$ and so due to $(24)$

$$
\frac{\Gamma_{i}\left(\theta_{u}\right)}{\Lambda_{1}(u)} \rightarrow 0
$$

for each $i, \theta_{u}<1$, and that $\epsilon$ is arbitrary.

\section{REFERENCES}

Adalakha, V. G. and Kulkarni, V. G. 1989. A classified bibliography of research on stochastic PERT networks. INFOR 27, 3, 272-296.

Crovella, M., Taqqu, M. S., and Bestavros, A. 1998. Heavy tails in the world wide web. In Practical Guide to Heavy Tails, R. Adler, R. Feldman, and M. S. Taqqu, Eds. Birkhauser, Boston, MA 2431.

Dembo, A. and Zeitouni, O. 1998. Large Deviations Techniques and Applications, Second ed. Springer, New York, NY.

Elmaghraby, S. E. 1977. Activity Networks: Project Planning and Control by Network Models. Wiley, New York, NY.

Embrechts, P., Kluppelberg, C., And Mikosch, T. 1997. Modelling Extremal Events for Insurance and Finance. Springer-Verlag, Berlin, Heidelberg, Germany.

Feller, W. 1970. An Introduction to Probability Theory and Its Applications, Third ed. Vol. 1. Wiley, New York, NY.

Heidelberger, P. 1995. Fast simulation of rare events in queueing and reliability models. ACM Trans. Model. Comput. Simul. 5, 1, 43-85.

HuAnG, Z. AND Shahabuddin, P. 2004. A unified approach for finite dimensional, rare-event Monte Carlo simulation. In Proceedings of the 2004 Winter Simulation Conference, R. Ignalls, M. Rossetti, J. Smith, and B. Peters, Eds. (Piscataway, NJ). 1616-1624.

Juneja, S. ANd Shahabuddin, P. 2002. Simulating heavy-tailed processes using delayed hazard rate twisting. ACM Trans. Model. Comput. Simul. 12, 94-118.

Juneja, S. AND Shahabuddin, P. 2006. Rare event simulation techniques. In Handbook on Simulation, S. Henderson and B. Nelson, Eds. Elsevier, Amsterdam, The Netherlands, 291350.

JuRECKova, J. 1981. Tail behavior of location estimators. Ann. Stat. 9, 3, 578-585.

Leland, W. E., TAqQU, M. S., Willinger, W., and Wilson, D. V. $1994 . \quad$ On the self-similar nature of ethernet traffic. IEEE/ACM Trans. Netw. 2, 1-15.

PAkes, A. G. 2004. Convolution equivalence and infinite divisibility. J. Appl. Prob. 41, 407424.

Petrov, V. V. 1975. Sums of Independent Random Variables. Springer-Verlag, New York, NY.

ACM Transactions on Modeling and Computer Simulation, Vol. 17, No. 2, Article 7, Publication date: April 2007. 
Pitman, E. J. G. 1980. Subexponential distribution functions. J. Austral. Math. Soc. Ser. A 29, 337-347.

SAdowsky, J. S. ANd BuckLew, J. 1990. On large deviation theory and asymptotically efficient Monte Carlo estimation. IEEE Trans. Inf. Theory 36, 3, 579-588.

Sigman, K. 1999. A primer on heavy-tailed distributions. Queu. Syst. 33, 261-275.

Received February 2004; revised December 2005 and August 2006; accepted October 2006 